\title{
Céréales, pains, levains et fours dans la région d'El Hoceima
}

Techniques alimentaires et notes sur des parlers arabes à la frontière de la berbérophonie

Cereals, breads, yeast and ovens in the region of El Hoceima: food techniques and notes on the Arabic spoken on the border with Berber-speaking areas

Dominique Caubet et Yildiz Aumeeruddy-Thomas

\section{(2) OpenEdition \\ Journals}

Édition électronique

URL : https://journals.openedition.org/ethnoecologie/3070

DOI : 10.4000/ethnoecologie.3070

ISSN : 2267-2419

Éditeur

Laboratoire Éco-anthropologie

Référence électronique

Dominique Caubet et Yildiz Aumeeruddy-Thomas, « Céréales, pains, levains et fours dans la région d'El Hoceima », Revue d'ethnoécologie [En ligne], Supplément 1 | 2017, mis en ligne le 17 octobre 2017, consulté le 19 novembre 2021. URL : http://journals.openedition.org/ethnoecologie/3070 ; DOI : https://doi.org/10.4000/ethnoecologie.3070

Ce document a été généré automatiquement le 19 novembre 2021.

\section{cc) (†)}

Revue d'ethnoécologie est mis à disposition selon les termes de la licence Creative Commons Attribution - Pas d'Utilisation Commerciale - Pas de Modification 4.0 International. 


\section{Céréales, pains, levains et fours dans la région d'El Hoceima}

Techniques alimentaires et notes sur des parlers arabes à la frontière de la berbérophonie

Cereals, breads, yeast and ovens in the region of El Hoceima: food techniques and notes on the Arabic spoken on the border with Berber-speaking areas

Dominique Caubet et Yildiz Aumeeruddy-Thomas

\section{Introduction}

1 La préparation des farines et des pains à partir de céréales, constitue une activité-clef en Méditerranée, zone de l'origine de la domestication des blés, de l'orge, du petit épeautre, du seigle et de l'avoine. Les céréales sont utilisées sous forme de semoules, de pâtes, de farines, de pains levés ou non levés depuis des temps anciens (Franconie et al. 2010). La nature des fours utilisés et les restes archéologiques de céréales retrouvés dans les fours ont permis de comprendre ce que représentait la cuisson des pains et dans certains cas les liens sociaux autour de leur fabrication (Samuel 1999).

2 Les travaux que nous présentons montrent des techniques alimentaires actuelles autour des céréales, notamment l'orge et le blé, jeunes non-matures, servant à la fabrication de farine de céréales grillées au four et de semoules. Nous nous sommes également intéressées au pain d'orge ou de blé fait avec la farine de moulin, à l'usage de levains traditionnels ainsi qu'à la place du four dans la sphère domestique.

Notre démarche transdisciplinaire ethnobotanique et linguistique s'attache aux discours et aux termes cherchant à mieux saisir les usages des différentes formes de parlers, et à traduire ce que les groupes sociaux rencontrés attribuent comme signification aux aliments préparés et à la nature des plantes qui les composent. Nos travaux se situent dans le Rif, région du nord du Maroc de l'ouest méditerranéen connue pour avoir été peuplée très anciennement et où l'agriculture témoigne jusqu'à nos jours d'une diversité remarquable de céréales. Les travaux des archéobotanistes 
montrent que les céréales et en particulier le petit épeautre et l'amidonnier (Triticum monococcum/T. dicoccum), le blé dur (Triticum durum, Triticum aestivum/durum), l'orge (Hordeum vulgare, Hordeum vulgare var. nudum) ainsi que les légumineuses telles que les lentilles (Lens culinaris), le petit pois (Pisum sativum) et la fève (Vicia faba) étaient présents ainsi que l'élevage d'animaux domestiques et la fabrication de poteries, 5500 à 5000 ans avant J.-C. (Morales et al. 2016). Les travaux d'ethnobotanique contemporaine menés par ces derniers montrent les techniques de fabrication des récipients alimentaires en argile ainsi que les modalités de stockage des céréales en pays Jbala (Peña-Chocarro et al. 2015). Hmimsa et Ater $(2008,2010)$ ont effectué une étude de l'agrodiversité rifaine et décrivent cette région comme un « hot spot » d'agrodiversité au Maroc. Ce sont les premières études mettant en relation diversité agraire, terminologies et appellations locales des espèces cultivées du Rif. Les études reliant les terminologies locales et la diversité des figuiers du Rif effectuées par Hmimsa et al. (2012 et 2017) et Aumeeruddy-Thomas et al. (2014a) mettent en lumière la richesse du vocabulaire agraire des paysans jbala concernant les variétés, la désignation des espaces agraires ainsi que des techniques de pollinisation complexe des figuiers. Ces travaux montrent des savoir-faire élaborés des paysans jbala. Les études ethnobotaniques et linguistiques dans le Rif ouvrent une voie pour mieux comprendre ces savoirs, à travers l'expression du langage et la nature des échanges entre communautés parlant des dialectes ou des langues différents.

Le Rif est en effet une situation idéale à la fois pour le linguiste et l'ethnobotaniste pour l'étude des situations d'échanges, de contacts de langues et de techniques alimentaires, aux frontières entre groupes arabophones et berbérophones. Les ethnobotanistes cherchent à comprendre comment ces différents groupes identifient leurs plantes, les utilisent et les nomment, leurs techniques alimentaires ainsi que les relations des gens entre eux à propos des plantes. Ils procèdent généralement à des séries d'enquêtes ouvertes autour de sujets ou de pratiques spécifiques associées à des catégories de plantes identifiées lors de différentes périodes de terrain. Les travaux conjoints d'ethnobotanique et de linguistique ont porté tout spécialement sur les céréales, le pain, le levain et les fours.

5 Pour les linguistes, il s'agissait de décrire des situations de contacts de langues entre le berbère et l'arabe marocain, ou darija, dans des régions très proches géographiquement, mais aux situations différentes:

- Taounil, village berbérophone, avec une informatrice dont l'arabe est langue seconde, - Msek, exemple rare d'un douar arabophone en situation minoritaire, entouré de villages berbérophones. Ses habitants se disent Rifains alors qu'ils ont l'arabe pour langue première.

6 En outre, il était important de revisiter des terrains rarement explorés, dans ce nordouest marocain, région méconnue, malgré le rôle clef qu'elle a joué dans l'histoire du pays. Elle a, en effet, connu des mouvements de population exceptionnels du fait de sa position géographique si particulière, à la limite de deux continents. Elle est le foyer des tout débuts de la présence de l'arabe, dès le viI/VIII siècle, dans ce qui est aujourd'hui le Maroc. Les parlers jbala-nord Taza font partie de ce que l'on considère comme la première couche d'arabisation du Maghreb, plusieurs siècles avant l'arrivée des Bédouins, du XI ${ }^{\mathrm{e}}$ au XIII ${ }^{\mathrm{e}}$ siècle.

7 Des études linguistiques ont été faites au début $\mathrm{du} \mathrm{xx}^{\mathrm{e}}$ siècle par deux travaux majeurs sur le Nord-Taza (Colin 1921) et la région d'Ouargha (Lévi-Provençal 1922). Des 
mémoires de licence ont été rédigés par des étudiants de Simon Lévy au début des années 90 (Abou El Haja 1995, Maghdad 1993, Khoukh 1993), mais ils sont restés inédits (voir Caubet 2017). Plus récemment de nombreux travaux ont porté sur les régions jbala plus occidentales, Anjra (Vicente 2000), Chaouen (Natividad 1998, Moscoso 2003, Ziamari \& Barontini 2008). Peter Behnstedt et Mostafa Benabou avaient commencé un travail d'atlas pour le nord-est du pays (Behnstedt \& Benabbou 2002, Behnstedt 2002, 2005 et 2007), mais les parlers arabes de la région d'El Hoceima n'avaient pratiquement jamais été décrits.

Dans le cadre du Groupe Jbala du CJB, en collaboration avec les Universités de Fès et Oujda, juste avant l'obtention d'un PICS (Projet International de Coopération Scientifique) La montagne et ses savoirs (IREMAM - Université de Fès), toute une série d'études ont été menées depuis 2012 dans le pays Jbala (Arsenne 2016, Naciri-Azzouz 2016, Caubet 2016, El Jettari 2017, Vicente et al. (Ed.) 2017)

\section{Matériel et méthodes}

Le travail présenté ici a été recueilli lors d'une mission conjointe d'ethnobotanistes et de linguistes effectuée dans le cadre du PICS La montagne et ses savoirs initié par l'IREMAM et du Programme Jbala du CJB (Centre Jacques Berque, Rabat) ${ }^{1}$ en février 2014. Outre D. Caubet et Y. Aumeeruddy-Thomas, y participaient Amal Maghdad, (traductrice à Tanger) et Khalid El Jettari (à l'époque étudiant en master 2 à l'Université d'Oujda). Outre cette mission conjointe Y. Aumeeruddy-Thomas a effectué plusieurs missions ethnobotaniques dans des zones arabophones du nord du Maroc, notamment dans le pays Jbala dans les communes de Bni Ahmed, Taounate et Ain Mediouna, Ouezzane, dont les données nous servent de point de comparaison dans ce papier. Elle travaille à ce sujet dans le Rif depuis 2006 sur l'agrobiodiversité rifaine notamment sur les arbres fruitiers (figuiers, oliviers et amandiers), les espèces céréalières et leurs plantes adventices ainsi que les légumineuses, avec des interrogations autour de leur domestication, des techniques agraires et alimentaires.

Nous étions logés dans un gîte rural du Parc National d'El Hoceima, situé dans le village de Taounil (Figure 1, flèche rouge) qui fait partie du groupe des Boqqoya. De là, nous nous sommes rendus dans le pays des Beni-Itteft, au douar Msek (Figure 1, flèche verte) ${ }^{2}$ pour revisiter le terrain effectué à l'été 1992 par Amal Maghdad pour son mémoire de maîtrise d'espagnol dirigé par Simon Lévy ${ }^{3}$. Ce mémoire avait également inspiré un premier travail effectué deux ans plus tôt par Khalid El Jettari ${ }^{4}$. 
Figure 1 : Extrait de la Carte Michelin du Maroc. 1. Flèche verte, emplacement du village de Taounil ; 2. Flèche rouge, emplacement du village de Msek

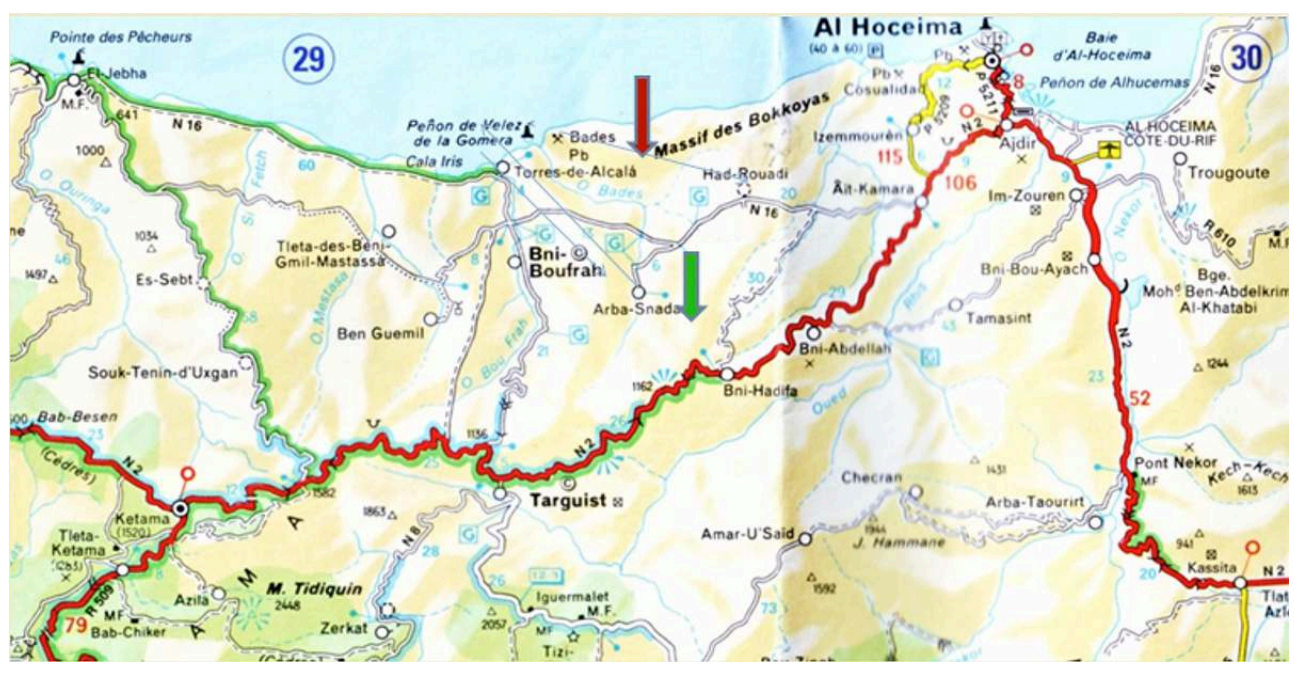

11 L'originalité de notre démarche réside dans le fait qu'elle est transdisciplinaire, fondée sur des enquêtes ethnobotaniques qui ont également permis le recueil de corpus linguistiques spontanés sur les parlers des enquêtés. Ces corpus ont permis d'apporter conjointement au linguiste une vision fine de l'articulation et des influences en cours entre parlers arabes et berbères, et des données ethnobotaniques inédites.

La carte publiée en 1932 par Renisio (Figure 2) dessine la frontière de la berbérophonie, en particulier pour la partie ouest du Rif qui nous intéresse ici. On remarque que nos deux terrains sont situés exactement sur cette frontière. Peter Behnstedt (2002) a travaillé sur ces frontières entre arabophonie et berbérophonie, plus à l'est (Beni Snassen et Nord Taza). Il se réfère au travail de Renisio pour sa partie, mais contrairement à nous - sur une zone certes petite - il a relevé des changements dans le sens d'une arabisation des zones berbérophones.

Sur une autre carte de Renisio (1932, Figure 2), Les Bni Itteft (trait vert) apparaissent avec la partie berbérophone qui correspond à la tribu des Aït Aïssa dont fait partie Msek, situé exactement à l'ouest de cette frontière. La Villa Sanjurjo correspond à l'emplacement actuel d'El Hoceima. 
Figure 2 : Les limites de la berbérophonie

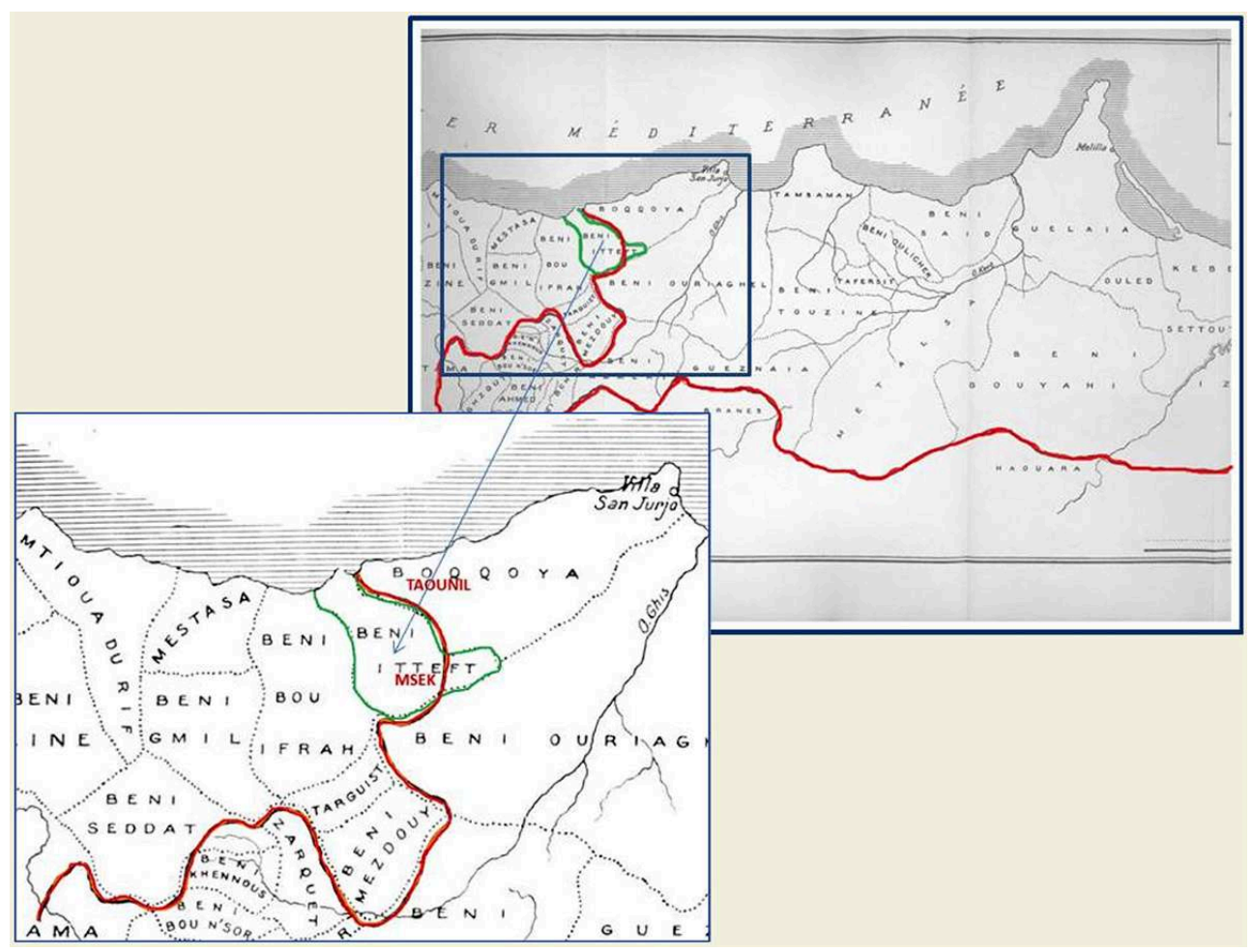

Trait rouge repris sur la limite proposée par Renisio. Le Bni Itteft (trait vert) avec une partie berbérophone qui correspond à la tribu des Aït Aïssa dont fait partie Msek, situé exactement à l'ouest de cette frontière. La Villa Sanjurjo correspond à l'emplacement actuel d'El Hoceima

Source : Renisio (1932)

En 1968, G. Maurer publiait une étude détaillée de géographie humaine sur ce qu'il appelait le «Haut Rif central » (Maurer 1968); il y dressait une carte des tribus où il apparaît clairement que Boqqoya et Bni Itteft en font partie, mais également des tribus situées plus à l'ouest comme les Bni Boufrah (voir Arsenne 2016). Les deux terrains examinés ici doivent être considérés comme rifains, quelle que soit leur langue maternelle, et faisant partie d'un même ensemble (Figure 3). 
Figure 3 : Extrait modifié d'une carte de Maurer (1968) (Communes rurales et fractions dans le Rif central)

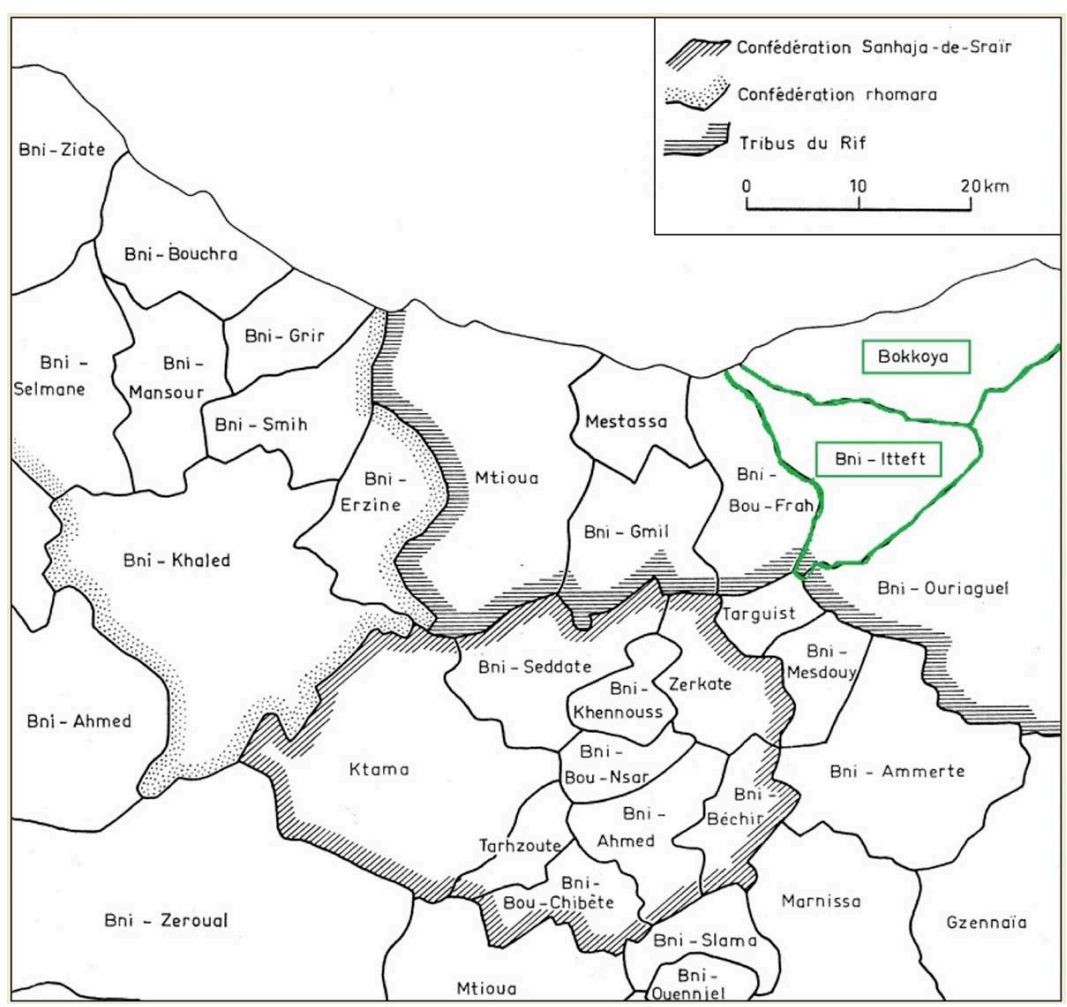

15 Nous allons présenter ces deux terrains qui diffèrent sur le plan linguistique et anthropologique, puisque situés dans des tribus différentes, avec des langues maternelles différentes.

À Taounil, notre informatrice était une femme de 50 ans, berbérophone de la tribu des Boqqoya, n'ayant pas fréquenté l'école et ayant appris l'arabe marocain dans le village même avec ses voisins originaires de Bni Itteft. Sa maîtrise de la darija n'est pas parfaite.

17 À Msek, il s'agissait d'une jeune femme arabophone d'environ 35 ans n'ayant pas non plus été à l'école. Le lieu-dit Sefri a une situation peu courante au Maroc, puisqu'il appartient à la minorité arabophone de la fraction berbérophone rifaine Aït Aïssa (ellemême minoritaire) de la tribu des Bni Itteft qui est majoritairement arabophone ${ }^{5}$. Cette situation minoritaire de la darija par rapport au berbère (ou amazighe), est suffisamment rare et peu décrite pour être soulignée.

Lors de cette mission, une enquête d'ethnobotanique a été menée par Y. AumeeruddyThomas, sur la culture des céréales, sur diverses préparations à base d'orge (zambu), et sur les techniques traditionnelles de la fabrication et de la conservation du pain et du levain dont nous allons rendre compte. Pour ce qui est de la dialectologie, ces entretiens nous ont donné accès à ces parlers arabes rifains marginaux, avec leurs traits pré-hilaliens, proches des parlers jbala, et en contact quotidien avec le berbère rifain. Il est à noter ici que ces traits pré-hilaliens, difficiles à relier directement à la situation des savoirs ethnobotaniques contemporains, constituent néanmoins une entrée importante pour une réflexion sur l'influence de possibles savoirs et savoir-faire ayant voyagé de l'est de la Méditerranée, l'arabisation elle-même provenant de l'est, rencontrant localement des savoirs et savoir-faire berbères. Pour des raisons de clarté 
sur le contenu scientifique des avancées d'une analyse conjointe ethnobotanique et linguistique, nous avons séparé ces deux groupes de résultats, étant bien entendu que les corpus communs aux deux constituent une première étape de cette collaboration qui nécessiterait une analyse des termes à plus grande échelle, méditerranéenne, d'est en ouest, et entre certains pays arabisés, tels que Malte, la Sicile en Italie et l'Andalousie en Espagne, pour répondre à nos questions concernant les transferts de savoirs à l'échelle méditerranéenne, un projet ambitieux que ces premiers résultats nous permettront possiblement de construire.

19 Sur le plan ethnobotanique, ces travaux nous ont permis d'identifier les chaînes opératoires de transformation comportant un lexique très riche, ainsi que l'importance culturelle associée aux produits céréaliers. Nous discutons cet ensemble de techniques et les usages des produits céréaliers dans le contexte de leur insertion au sein des champs céréaliers et vergers d'amandiers environnants, et dans l'unité domestique. Pendant notre séjour nous avons profité des enquêtes pour développer une compréhension plus englobante du système alimentaire des groupes étudiés et à ce sujet, avons aussi enquêté sur les plats mangés en début de printemps, période pendant laquelle nous avons effectué une mission conjointe. Nous ferons ainsi référence à l'usage des herbes de cueillette "sauvages " y compris des pousses d'asperges, plats de saison qui ont été partagés avec nous. Nous faisons ici appel à la méthode très classique en anthropologie qui est celle de partager les plaisirs de la table avec nos hôtes afin d'accéder et de comprendre ce que manger veut dire dans les deux villages où nous avons mené nos enquêtes.

\section{Résultats}

\section{Taounil : La préparation de zəmbu à base de farine d'orge jeune et les asperges}

Le village est situé dans le Parc National d'El Hoceima, sur une piste neuve menant à la ville mythique de Badis (Figure 4). Nos enquêtes auprès de F. notre hôte, portent sur des entretiens enregistrés menés chez elle notamment sur le pain et zəmbu, un aliment préparé à base de farine d'orge jeune. Nous avons continué cette enquête avec deux sorties sur le terrain avec elle où elle nous a montré les différents jardins, les techniques de stockage des céréales, les lieux de cueillette des asperges sauvages ainsi que des herbes « sauvages » consommées au printemps. 


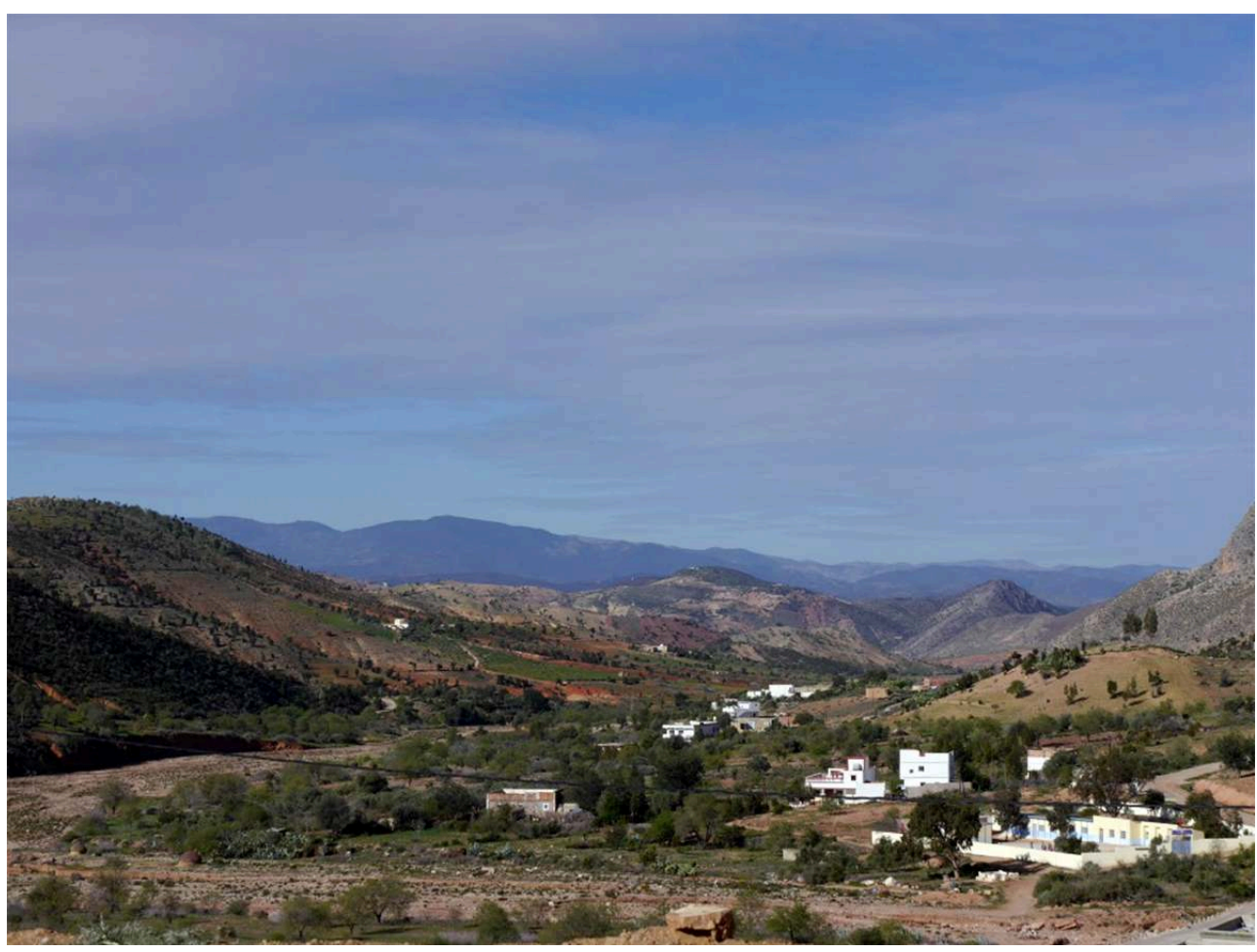

(C) Y. Aumeeruddy-Thomas

\section{Corpus}

Zəmbu : textes recueillis, enquête ethnobotanique Y. A-Thomas, transcription linguistique D. Caubet

Notre informatrice explique :

(Texte 1) hā $\underline{d i}$ (zəmbu) kayāklū-ha, hayda, kayEəžnū-ha b-əl-ma sxūnīn u kayāklū-ha...

Ça, c'est zembu (farine tamisée) ; ils la mangent comme ça, juste en la pétrissant avec de l'eau chaude et ils la mangent.

22 Sur l'enregistrement 1 (fichier sonore 1), elle poursuit :

(Texte 2) āhi b-əz-zabda, āhi b-əz-zit, āhi b-lasol, lli bg̀iti, lli bg̀iti ntīna...

Soit avec du beurre, soit avec de l'huile, soit avec du miel, comme tu veux...

Elle montre la différence avec les grains épais restés dans le tamis, dšǐ̌a :

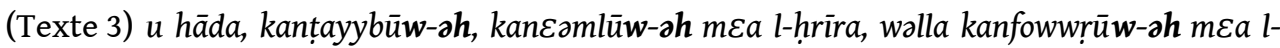
lbon walla kanțayybūw-oh hayda, kayəntkal hayda, kayEəmlu l-u z-zìt u kayāklūw-oh et celui-là, on le fait cuire, on le met dans la hrira ${ }^{6}$, ou bien on le fait cuire à la vapeur (et on le mange) avec du petit lait ou bien on le fait cuire tout seul, en y ajoutant de l'huile pour le manger.

Ce média ne peut être affiché ici. Veuillez vous reporter à l'édition en ligne http:// journals.openedition.org/ethnoecologie/3070

À la fin du XIX ${ }^{e}$ siècle, Mouliéras venu d'Algérie à la découverte du Maroc Inconnu (1895 : 65), décrit le Nord du pays avant le colonialisme. Il y a consacré deux tomes et dans la 
deuxième partie qui porte sur les 'Djebala', il évoque le zambu chez les Beni Mansour (Ghomara) (1899: 279) : «La nourriture habituelle des Beni-Mençour ne varie guère ; c'est l'inévitable biçar, le zambou d'orge, l'arguel', le maïs. On les réduit en farine, on détrempe cette farine dans de l'eau froide et on la mange avec les doigts en en faisant des boulettes. ». Un peu plus loin (1899: 519), il parle la consommation de dšiša à l'occasion de ce qu'il nomme 'le premier de l'an païen' : « Ils ont également conservé à travers les âges le souvenir confus du premier de l'an du calendrier païen, le $1^{\mathrm{er}}$ Iennaïer (janvier), comme ils disent, et, chose bizarre, ils ont une façon spéciale de le célébrer: pendant cette journée-là, ils avalent des quantités invraisemblables de dchicha, semoule grossière qu'ils font cuire dans l'huile. (...) »

Amal Maghdad questionne F. sur la façon de manger la dš̌̌ša :

kayontkal hayda?

Ça se mange comme ça?

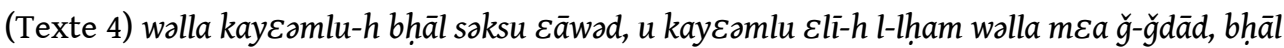
soksu

ou bien, on peut aussi le préparer comme le couscous, avec de la viande ou du poulet, comme le couscous.

À ce propos, M. Monkachi (1997: 96) explique :

« Le couscous est, avec le pain, l'aliment de base. De blé ou d'orge, le couscous est de la graine de semoule cuite à la vapeur. Il peut être accompagné d'un bouillon de légumes et de viande. »

\section{Tasukkant " les asperges sauvages "}

Le mot ta-sukkan-t a la forme d'un substantif féminin en berbère. Les asperges sauvages sont appelées səkkum (əl-bərr) en marocain, comme le confirment Mercier (1951 : 182) et Iraqui-Sinaceur $(1993,4: 831)$ dans leurs dictionnaires. Mercier donne : "Sekkum, asperge »; Colin : « səkkūm, n. coll. Bot. Asperges sauvages ».

Salem Chaker confirme que la racine existe en Berbère (mais pas en rifain) et en arabe maghrébin. Il explique le passage de sekkum à ta-sukkan-t par le fait que $m$ devient $n$ devant le suffixe $-t$ par assimilation à la dentale qui suit ${ }^{8}$.

(Texte 5) katənbət hayda f-əl-aṛd, hìya fi-ha š-šukk, bəllāti, hìya gìir hnāya fūq-d-dār dyāl-na, hìa katkūn gìị f-hād-əl-waxt (weqt), ka-tənbət b-wahd-a.

Ça pousse comme ça dans la terre; ça a des épines, attends, tu peux en trouver juste à côté au-dessus de la maison, ça ne pousse qu'à cette période de l'année, ça vient tout seul.

Dans l'enregistrement 2 (fichier sonore 2), F. explique :

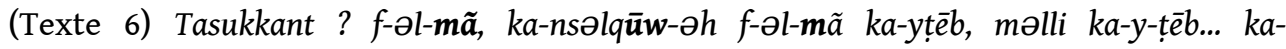

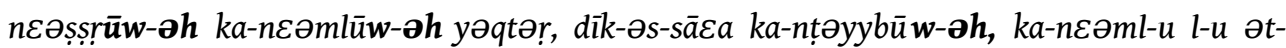

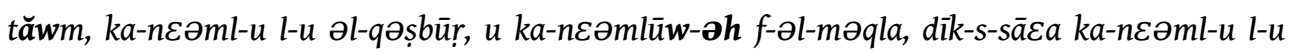
əl-băyțāt, dik-əs-sāea, ka-yə... ka-yəntkəl.

Les asperges? Dans l'eau, ça se fait bouillir dans l'eau, et quand c'est cuit, on les presse, on les laisse s'égoutter et après on les prépare, on ajoute de l'ail, de la coriandre, puis on les met dans la poêle et on ajoute des œufs et après, on... on les mange.

Ce média ne peut être affiché ici. Veuillez vous reporter à l'édition en ligne http:// journals.openedition.org/ethnoecologie/3070 


\section{Taounil : Analyse linguistique à partir des textes 1 à 6}

On remarque quelques traits caractéristiques qui en font un parler assez particulier, sur le plan phonétique, morphosyntaxique et lexical, avec des traits spécifiques du Nord et d'autres qui le rapprochent du Maroc central.

Pour ce qui est du lexique, l'utilisation du verbe $\varepsilon m a l$ 'faire', de l'adverbe hayda 'comme ça' (au lieu de hakda), ou de ğ-ğdād pour désigner le poulet (au lieu de $d$-džăž) ; ce qui est la norme au Nord du Maroc.

On a également des confusions dans les accords en genre. Des noms masculins accordés au féminin et vice-versa : zəmbu qui est masculin en arabe, est accordé au féminin (démonstratifs et pronoms compléments) par notre informatrice, mais elle n'est pas locutrice native :

hādi (fém), 'celle-ci' ; kayāklū-ha (fém) hayḍa, 'ils la mangent comme ça'; kayعəžnu-ha (fém)b-ol-ma sxūnin u kayāklū-ha (fém), 'ils la pétrissent avec de l'eau chaude et ils la mangent'.

On remarque l'influence du substrat berbère ci-dessus avec un accord du substantif masculin singulier al-ma 'l'eau' avec l'adjectif pluriel sxūnin 'chauds'. En effet, en berbère, l'eau est un pluriel aman [litt. les eaux].

L'influence est également sensible dans la réalisation nasalisée de la voyelle /a/ à la

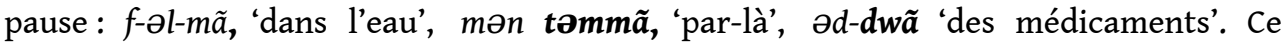
phénomène connu pour le kabyle a été décrit pour le rifain par Cadi $(1987: 29)$ et Lafkioui $(2007: 13)$.

38 On trouve des diphtongues ăw et ăy, dans des termes comme : ət-tăwm, əl-băyțăt, əl-lăwz 'ail, œufs, amandes'. On remarque que le /ḍ/ réalisé [ț], dans le mot əl-băyțāt 'les œufs'. Enfin le /q/, réalisé $[\mathrm{x}]$ dans le mot əl-waxt 'le temps'. Tout ceci existe aussi dans les parlers du nord.

Il y a de nombreuses spirantisations des dentales et des bilabiales, /d/ réalisé [d] et /b/

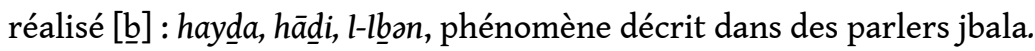

Et enfin, une forme verbale, rapproche ce parler des parlers du Nord du Maroc, la $1^{\text {ère }}$ personne du pluriel kantayybu, 'nous faisons cuire' ou kancamlu 'nous faisons' se trouve allongée, 'épaissie' lors de l'adjonction du pronom complément de $3^{\text {ème }}$ personne singulier $-\mathrm{h}$. Au lieu de kanțayybū- $h$ 'nous le faisons cuire' ou de kanEamlū- $h$ 'nous le faisons', on a toute la série de formes où $\bar{u}-h>\bar{u} w-\partial h$, de l'exemple (3) : kanțayybūw-əh,

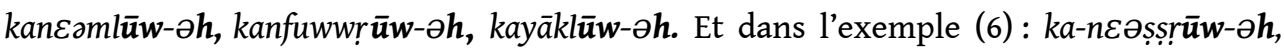
$k a-n \varepsilon \partial m l \bar{u} w-\partial \boldsymbol{h}, k a-n t \ni y y b \overline{\mathbf{u}} \boldsymbol{w}-\partial \boldsymbol{h}, k a-n \varepsilon \partial m l \overline{\mathbf{u}} \boldsymbol{w}-\partial \boldsymbol{h}$.

1 Enfin, on note l'amuïssement du $h$ dans les pronoms affixes où -ha est réalisé $-a: b$ wahd-a 'par elle-même' ou kaycəml-u l-a 'on lui met'.

Nous résumons les traits particuliers de ce parler dans les tableaux 1 et 2. 
Tableau 1 : Traits phonétiques

\begin{tabular}{|c|c|}
\hline & Taounil \\
\hline lal & $/ ə /$ réalisé $[œ]$ H-lhœm \\
\hline Réalisation $\mathrm{du}-a$ à la pause & Nasalisation à la pause: $f$-əl-mæ?ֶ, 'dans l'eau' mən təmmæ? 'par-là' \\
\hline Diphtongue ăw et ăy & ət-tăwm, əl-băyțāt, əl-lăwz 'ail, œufs, amandes' \\
\hline Iq & [q], réalisé $[x]$ dans le mot $\varrho /$-waxt 'le temps' \\
\hline$/ t / / \mathrm{d} / / \mathrm{d} /$ & Id / réalisé [t] əl-băytăt 'les œufs' \\
\hline $\begin{array}{l}\text { Spirantisations: } \\
\text { /d/ réalisé [d] } \\
\text { /b/ réalisé [b] }\end{array}$ & $\begin{array}{l}\text { hāyda, hãdi } \\
\text { I-lbonn }\end{array}$ \\
\hline Iž/ réalisé [ğğ] géminé & Gémination žž > [ğğ] ğğğdād \\
\hline amuïssement du $/ \mathrm{h} /$ dans les pronoms personnels de 3ème personne & $\begin{array}{l}\text { Pas toujours, mais } b \text {-wahd-ha >b-wahd-a 'par elle-même' ou } \\
\text { kayzəml-ul-a 'on lui met' }\end{array}$ \\
\hline
\end{tabular}

D. Caubet

Tableau 2 : Quelques points de morphosyntaxe

\begin{tabular}{l|l}
\multicolumn{1}{c}{} & \multicolumn{1}{c}{ Taounil } \\
\hline Le préverbe de l'inaccompli & ka- \\
\hline Le présentatif $\boldsymbol{d}$ & Non \\
\hline Pronom indépendant de 2ème personne & Ntĩna \\
\hline Accords de mots sing. au pluriel (infl.berbère) & el-ma sxünĩn
\end{tabular}

D. Caubet

\section{Taounil : Analyse ethnobotanique}

\section{Le pain et le four}

Le pain préparé par notre hôte nous est présenté dès notre rencontre comme un pain ordinaire $(\varepsilon \bar{a} d i)$; elle veut dire par là un pain fait maison à base de farine d'orge. Elle fait simplement remarquer qu'il est meilleur que celui acheté au souk et qu'elle le fabrique elle-même. En effet, sa maison, comme toutes les maisons rifaines possède un four qu'elle nomme aynur, situé à l'extérieur. Étant donné la saison (fin Février et début Mars), notre conversation a été rapidement orientée vers la farine du moment, zambu.

Zambu se fait avec de la farine d'orge jeune (au stade précédant la maturité et la récolte), grillée, pilée, puis tamisée, le tamisage donnant une farine fine appelée zambu. La partie non tamisée, plus grossière est nommée dšiša et fait l'objet d'une préparation similaire au couscous (voir plus haut la citation de Mouliéras 1899: 519 et 279). Le panier dans lequel on recueille zəmbu après le tamisage est appelé andu (Figure 5) 
Figure 5 : Le panier dans lequel on recueille zembu après tamisage est appelé andu. Il est fabriqué en paille de waduz ou dum (Chamaerops edulis)

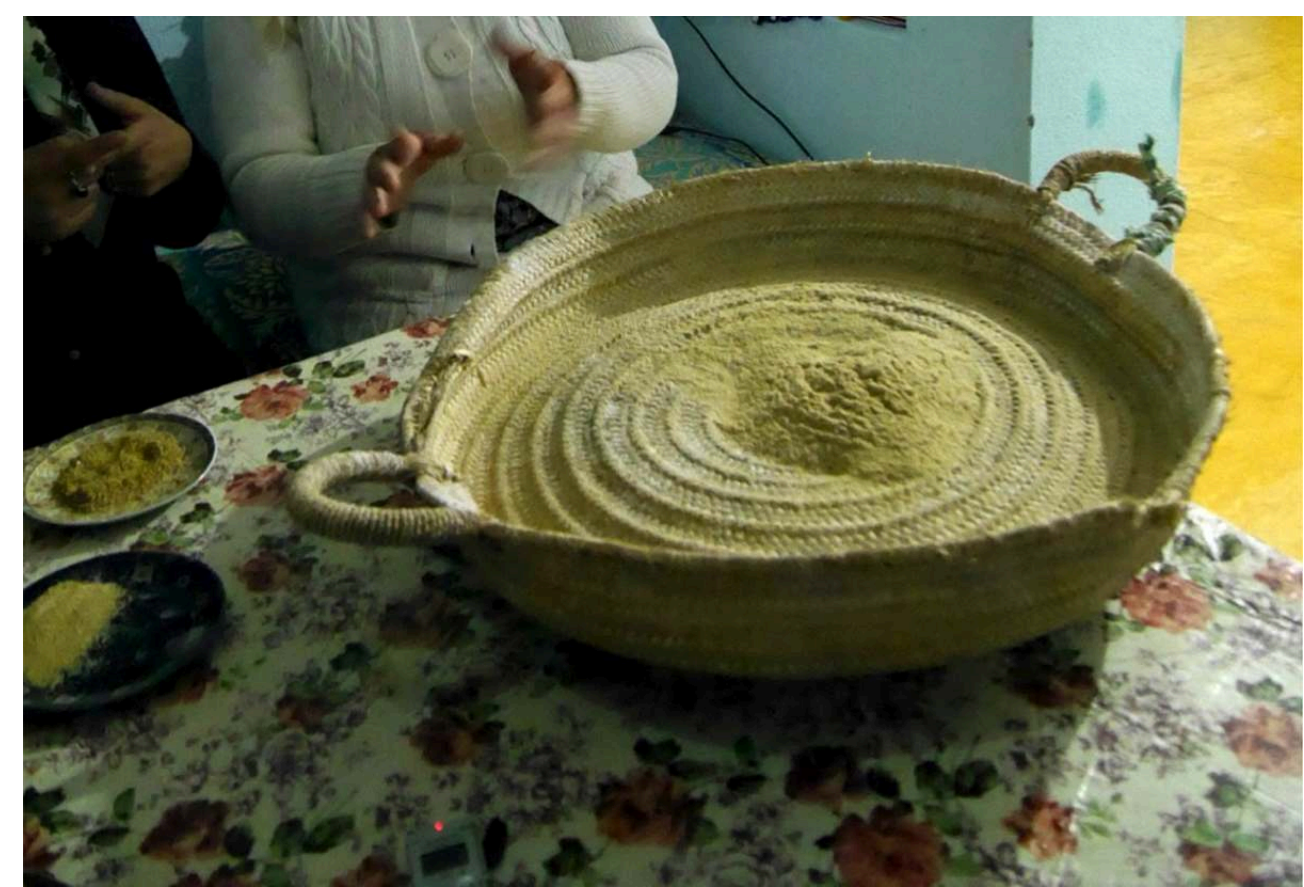

(C) D. Caubet

\section{Zambu chez les Boqqoya}

À Taounil, notre hôte nous explique que les Boqqoya appellent zambu en berbère tazemmit imermez (zombu d'orge) pas mûr cueilli pendant 'le mois 4', c'est-à-dire en avril. Le zambu de blé ou la-qmah (blé) arrive plus tard en été après l'orge. C'est celui qu'on mange avec les fruits de hindiya, le figuier de Barbarie que l'on trempe dans cette farine très fine et gouteuse ; son goût étant semble-t-il associé au fait que les graines sont légèrement grillées.

En effet, les graines d'orge cueillies peu avant la maturité sont déposées dans le four après avoir cuit le pain, une fois le feu éteint. Après une nuit de séchage au four, les graines sont déposées sur une natte pour finir de sécher au soleil. Elles sont ensuite pilées dans le maḥraz, pilon fait d'un bois très dur, le EarEar (Thuya sp.) très utilisé dans cette région notamment dans les anciennes maisons en bois. Le bâton qui sert à piler est fait de bois de lūz (amandier)

47 Le zambu d'orge est mangé selon la préparation décrite ci-dessous (Figures 6, 7 et 8). Nous l'avons dégusté au petit-déjeuner. Au moment de le servir, on pétrit la farine d'orge jeune tamisée avec de l'eau chaude pour former une pâte, découpée en morceaux grossiers :

48 Cette farine est très prisée y compris par des gens qui ne sont pas présents, pour lesquels elle peut être préparée et ensuite expédiée. Elle fait partie de toute une gamme de produits de printemps qui contribuent au bol alimentaire des habitants de Boqqoya. 
Figure 6 : Zəmbu, étape 1

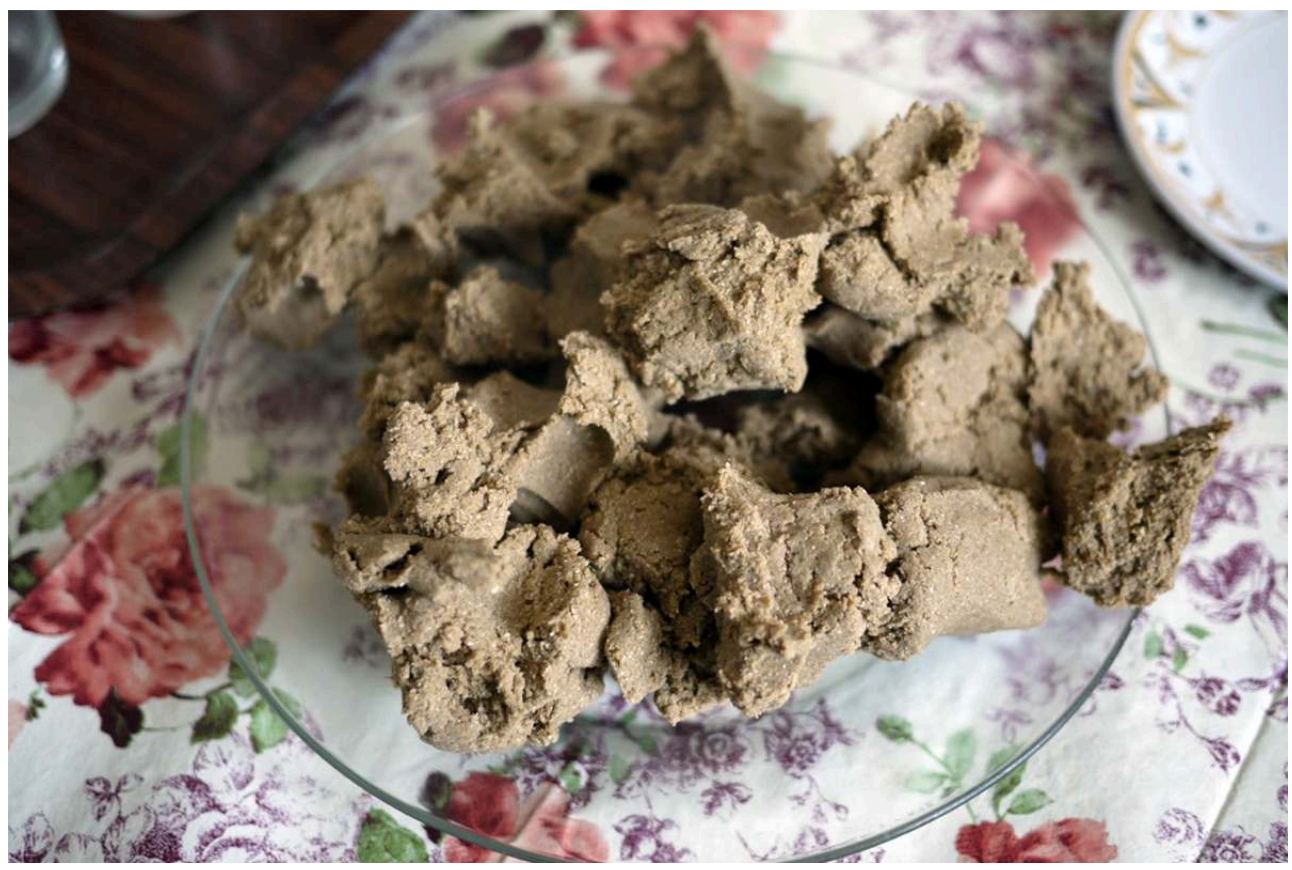

(c) D. Caubet

Figure 7 : Zəmbu, étape 2 : on forme une boule dans le creux de la main

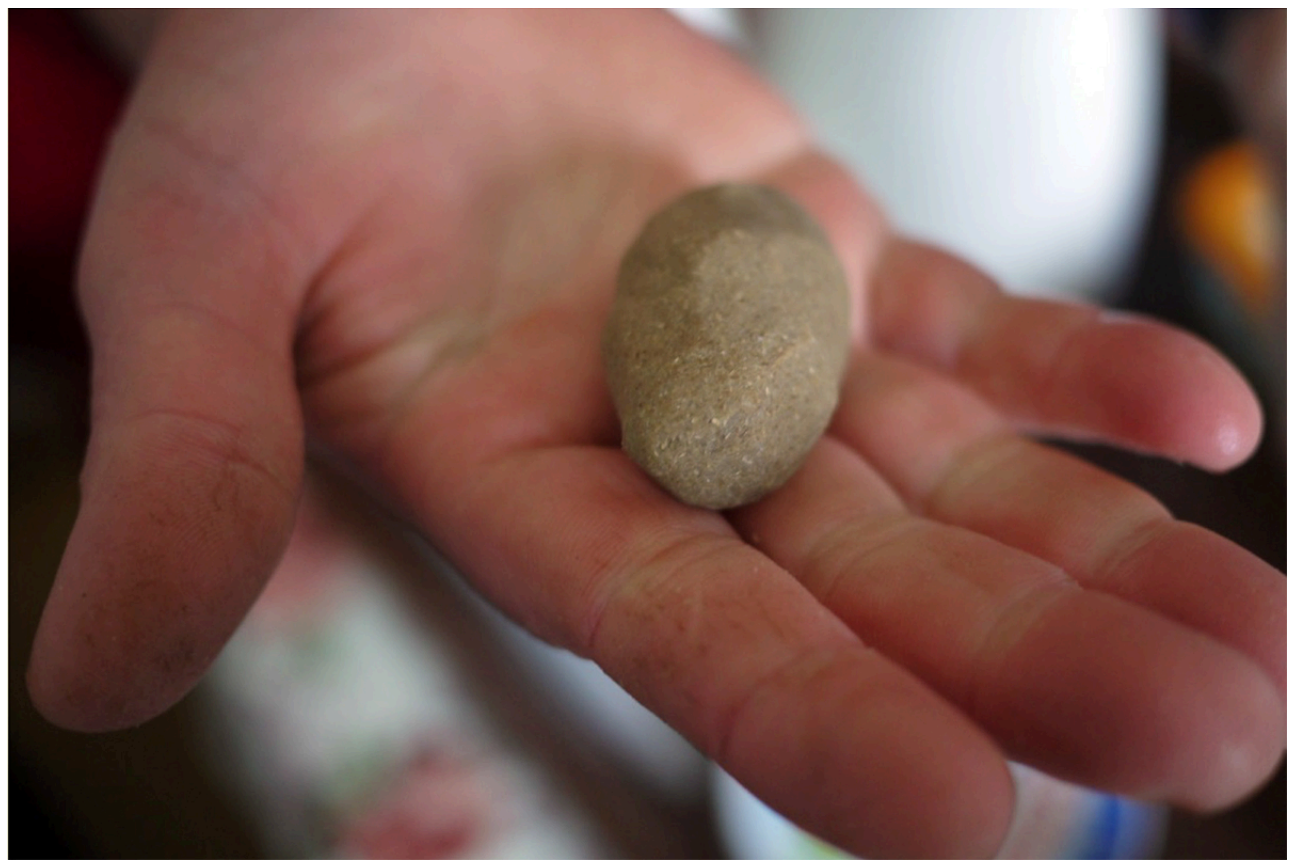

(c) D. Caubet 
Figure 8 : Zəmbu, étape 3 : on la trempe dans de l'huile d'olive

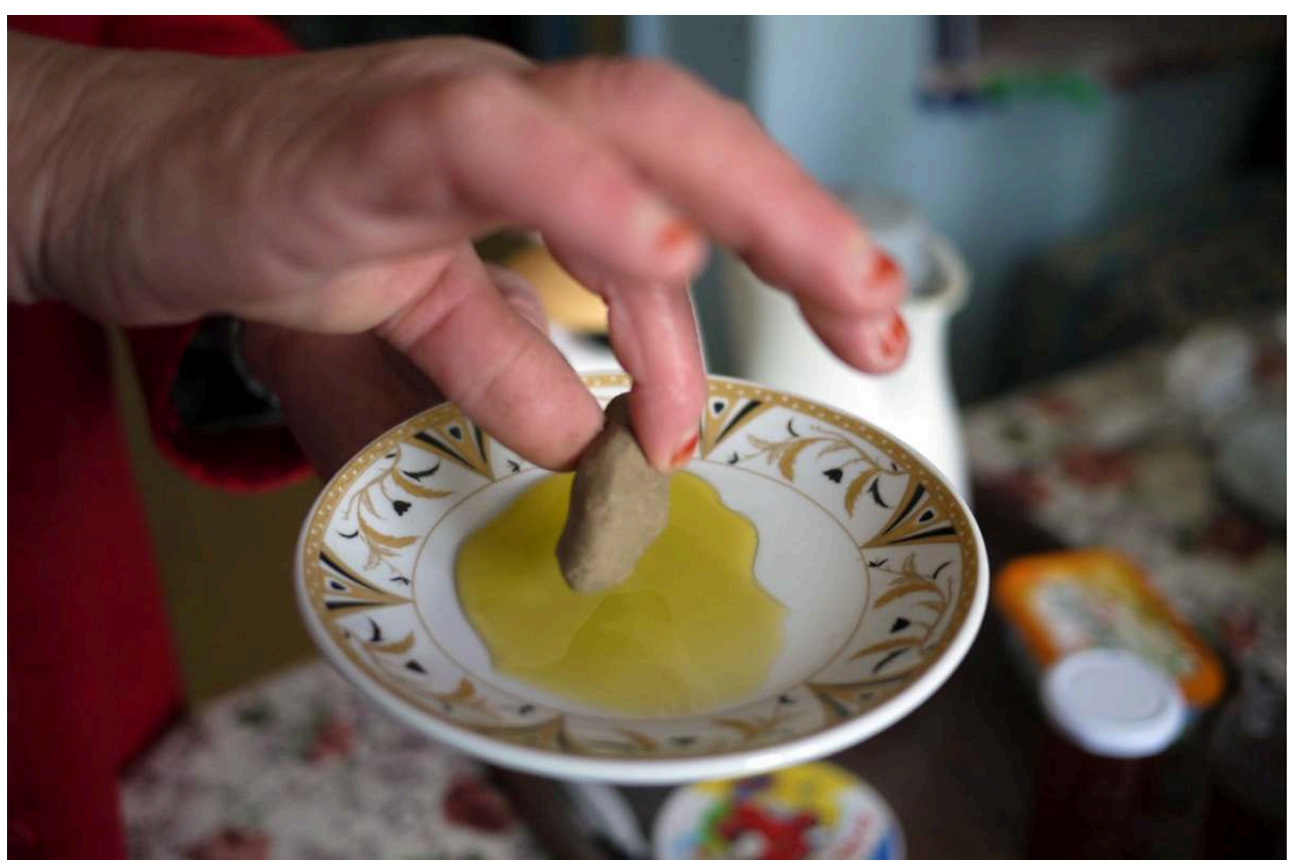

(c) D. Caubet

\section{Zombu et/ou zommēta, dans le Rif et dans d'autres régions du Maroc et de méditerranée}

L'usage de zambu au Maroc est connu au moins depuis le XIII ${ }^{\mathrm{e}}$ siècle. Rosenberg (1980 : 493) parle d'une préparation « d'orge dont le grain encore en lait donne une sorte de farine (azenbo) (...)» Il cite également la cașida (1980 : 484) « Dans le Rif, aux XIII et XIV siècles, c'est de l'orge légèrement grillée, moulue et cuite dans l'eau » et dit dans la note 52 (1980 : 499) que "Ce mets très simple, rapporté selon la tradition au calife 'Umar, passe pour avoir la baraka».

La préparation de zambu et ou son usage tels qu'observés à Taounil présentent des similitudes et des disparités avec ce que nous avons observé ailleurs en pays Jbala, plus à l'Ouest et avec les diverses formes de préparation de ce type de farine ailleurs au Maroc. Rappelons que les Boqqoya sont des Rifains amazighes et non des Jbala, bien que de nombreux traits sociaux et linguistiques soient communs à ces deux groupes.

51 Les Jbala arabophones utilisant des parlers arabes pré-hilaliens du Rif Central, tels que les Bni Ahmed, ou du Pré-Rif (versant sud du Rif) tels que les habitants de Ouezzane, Ain Mediouna, ou alors les groupes bédouins frontaliers des Ain Mediouna tels que les Hyayna, utilisent et connaissent tous cette forme de préparation de l'orge jeune.

52 Chez les Bni Ahmed, zambu est toujours fabriqué à partir de la farine d'orge récoltée de façon prématurée, également grillée et transformée en une farine fine utilisée pour tremper des figues fraîches. Nous l'avons mangé avec des figues de printemps, l-bākōr, figues fleurs gorgées d'eau que l'on ouvre et que l'on trempe dans cette farine fine et délicieuse. l-bākōr, la figue fleur est en soi un signe de baraka car ne nécessitant aucune intervention humaine pour sa pollinisation. (Observations Y. Aumeeruddy-Thomas et Y. Hmimsa) 
53 À Ouezzane, on trouve les deux noms, zambu et zammêța. Les habitants de la ville d'Ouezzane disent que zəmbu est le mot utilisé par les gens des montagnes et zəmmèța par les gens de la ville; ils laissent entendre ainsi qu'ils seraient de la plaine. Nous avons aussi relevé l'usage de zəmmețta chez les Hyayna, une tribu où prédominent des parlers d'origine bédouine, mais également à Ain Mediouna, village jbala situé en zone frontière les Hyayna. Nos travaux montrent que les échanges matrilinéaires sont fréquents entre ces deux groupes Jbala et Bédouins Hyayna, les deux parlant néanmoins des parlers arabes très distincts (Aumeeruddy-Thomas et al. 2014b).

Dans d'autres régions du Maroc, zammēta, ou zammita désigne une préparation toute autre, qu'on appelle aussi sallu, et composée de nombreuses farines toujours issues de graines prématurées grillées et pilées. Plusieurs recettes trouvées en ligne associent blé, graines de pastèque, graines de citrouille, pois chiche, graines de fenouil, d'anis, de lin et épices ${ }^{9} ;$ d'autres y mettent aussi de l'orge ou du millet ${ }^{10}$. Sollu est une préparation de fête, composée de farine de blé, d'amandes, de sésame, de beurre et d'épices essentiellement ${ }^{11}$. Zammețta dans le Moyen Atlas (Ifrane) est fabriqué à partir de farine d'orge jeune grillé auquel est rajouté du beurre, de la pâte de sésame et d'amandes écrasés, à mi-chemin entre zambu et sollu. Dans tous les cas, ces recettes contiennent de la farine de céréales prématurées (ou jeunes) grillées.

Selon Aubaile-Sallenave (1999), cette préparation est répandue dans toute la Méditerranée musulmane. Dans ses travaux sur la nourriture des accouchées, elle note que ce plat est préparé le septième jour suivant la naissance du bébé ou lors du Mouloud (date de la naissance du Prophète). Elle a identifié des préparations semblables, dont la tommina utilisée dans la Mitidja, près d'Alger (Desparmet 1918: 127 cité par Aubaile-Sallenave). Au Maroc, toujours selon Aubaile-Sallenave citant les travaux de Mas 1959 et Mathieu et Maneville 1952, cette préparation se nomme à Rabat, sello, slilu à Fès, slîlo ou tqâut à Marrakech (selon la transcription des auteurs). Elle rapporte en outre que dans ces préparations, à la farine d'orge grillée s'ajoutent des amandes grillées et pilées avec du beurre fondu et des aromates, graines de fenouil, d'anis vert, de sésame, cannelle, préparation destinée principalement aux accouchées. Elle est aussi appelée azenf à Sefrou concernant une préparation très semblable à sollu ; azenf se compose de farine grillée, miel, beurre et sésame (Mas 1959 cité par AubaileSalenave 1999, tout comme aznef chez les Ait Seghrouchen (Destaing 1920 cité par Aubaile-Salenave 1999).

Selon nos observations, ces mélanges ressemblent aussi étrangement à l'amlu préparé dans les régions de l'arganeraie, amlu étant alors une pâte faite d'amandes pilées torréfiées, d'huile d'argan, de beurre et de miel, devenu un produit de terroir très populaire avec la reconnaissance à l'échelle internationale des propriétés de l'huile d'argan.

Plus à l'Est, en Libye, zummîta est une préparation à base de farine d'orge (voir Pereira 2012 pour l'Ouest du pays à Jebel Nefusa). Dans la note 9, Pereira cite Gioia Chiauzzi, la personne qui a recueilli le corpus analysé, en expliquant qu'elle « a pu enregistrer une interview qu'elle a conduite à Jadu auprès d'un agriculteur. Elle enquêtait alors sur les rites agricoles et notamment sur l'importance du rôle de l'intervention des marabouts locaux. ». Pereira poursuit en disant que «L'auteur précise que la zummîta est un plat typique du Žébel qui requiert une longue préparation. On sèche au soleil des grains d'orge mondés et pilés, puis on les tamise en y ajoutant des épices, tel que le cumin. On obtient ainsi une farine que l'on nomme swîga. Une fois pétrie avec de l'eau froide, on y 
ajoute de la graisse de mouton. Cette préparation est adoucie avec du miel, du sucre ou des dattes. C'est la pâte ainsi obtenue qui s'appelle zummita (note 6, p. 851). »

\section{Asperges et plantes adventices du printemps}

Outre le zambu d'orge ou šEir qui est une nourriture de printemps, nous présentons d'autres nourritures du printemps qui caractérisent le bol alimentaire des habitants du Rif et que nous avons observées chez les Boqqoya. La cueillette des asperges "sauvages", tasukkant (Asparagus spp. Voir Figure 9) et de leur transformation sur le plan culinaire ont été suivis pas à pas lors de l'enquête ethnobotanique (voir textes 5 et 6). Les Boqqoya, nomment aussi les asperges en berbère izilfen. La technique alimentaire qu'ils emploient pour préparer les asperges consiste à les faire bouillir rapidement et ensuite à évacuer l'eau de cuisson. Notre hôte cuisine ensuite ces asperges en omelette tout comme l'omelette aux asperges sauvages du printemps aussi fabriqué sur la rive Nord. Le caractère strictement sauvage de cette plante est cependant discutable. Bien qu'elle ne soit pas plantée, elle est protégée en terrain agricole, dans les champs et dans les haies. À ce titre tasukkant peut être comparée à un grand nombre d'espèces adventices favorisées par l'homme dans des espaces domestiques fortement sous l'emprise de l'araire ou dans des haies ou chaque élément a un usage, outre celui de former des frontières entre parcelles agricoles. Bien que ces pieds ne soient pas plantés, ils ont été protégés. La cueillette d'asperges se fait ainsi dans des terrains agricoles où poussent des oliviers, des amandiers et des céréales. Les pieds d'asperges sauvages sont connus de la cueilleuse qui sait exactement où les trouver tout au long d'un périple de cueillette qui la fait visiter son jardin personnel, qu'elle nomme en arabe le-ḩwānāt et en berbère, Tigzdant. Elle parcourt également des parcelles agricoles appartenant à d'autres membres de la famille. La cueilleuse ne s'aventure pas en terrain inconnu et c'est bien dans un territoire appartenant à des membres de sa famille ou de ses voisins avec qui elle échange régulièrement qu'elle peut cueillir sans avoir à demander la permission. 
Figure 9 : Cueillette de tasukkant 2014

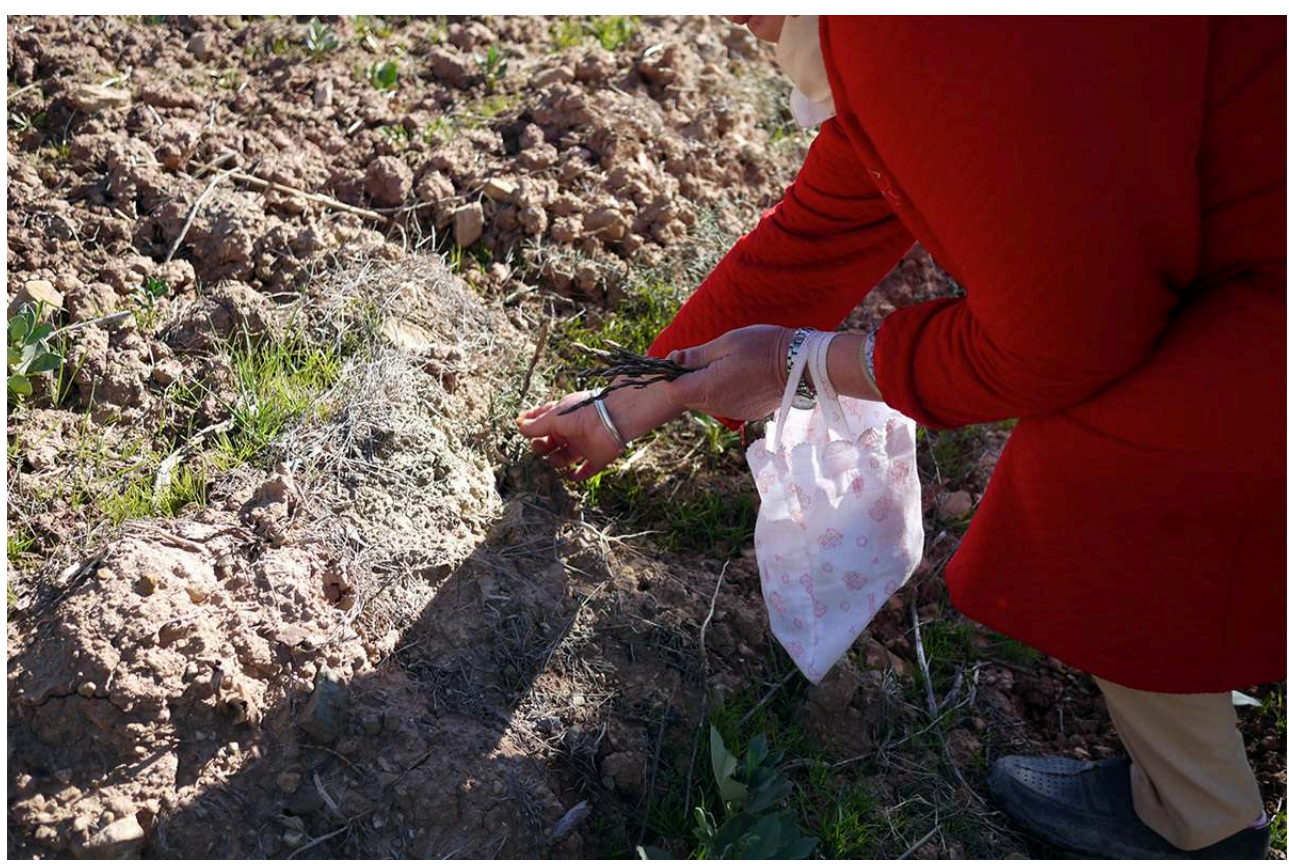

(c) D. Caubet

$\mathrm{Au}$ cours de ce périple, elle montre un grand nombre de plantes adventices, certaines comestibles pour l'homme, tels que karnūšs (Arisema sp.) petite liliacée dont les tubercules sont consommés en temps de disette, xaršŭf baldi (artichaut qui pousse tout seul, ou parfois planté). Il s'agit d'une variété locale d'artichaut dont les tiges sont très prisées pour des tajines et qui s'oppose au xaršūfa romiyya l'artichaut cultivé. Notre hôte énumère ainsi une grande diversité d'espèces herbacées, toutes connues localement pour être utilisées dans la Baqqula (cf. Clochey et Aumeeruddy-Thomas, ce volume), Abithes, Gernina (chardon sauvage), sirga (blettes sauvage), tašzint beldi (en berbère), l'ortie locale qui ne pique pas, par opposition à de grandes orties dites imodiš romi qui piquent. Ce sont des plantes connues et consommées à travers le Maroc et dont les vertus dépuratives sont connues et les plats préparés fort appréciés au printemps.

Des buissons comme fazgart (arabe) ou azarin (berbère) (Ziziphus sp.), sont conservés bien que poussant spontanément dans des terrains agricoles ou dans des friches. Les fruits de azarin, azarin n'zugart sont vendues chez l'épicier. Au passage elle précise que llūz morr, l'amande amère est récoltée et vendue 20 MAD (dirham marocain, environ 10 dirhams $=1$ euro) au marché comparé à l-lūz $\varepsilon \bar{a} d i$ (amandes normales, douces) qui se vendent 50 MAD.

61 Comme les asperges, diverses plantes de ces jardins poussant spontanément semblent avoir un statut qualifié localement de tasukkant boldi. Selon Delpancke et AumeeruddyThomas (ce volume), la notion de baldi est cruciale dans la distinction entre ce qui est valorisé car provenant du lieu et romi appartenant à d'autres régions ou venant d'ailleurs. Dans le système alimentaire au Maroc, le boldi est très valorisé car il s'oppose à ce qui est industriel et chimique (Simenel 2010). L'exemple par excellence au Maroc de cette différenciation entre romi et baldi étant les poulets romi, poulets de batterie qui s'opposent au poulet baldi. 
62

\section{d'autres parties du Rif arabophone ainsi qu'à Ceuta de passer allègrement du darija au berbère, montrant ici comment s'opèrent les échanges liés à la forte mobilité des gens à travers le Rif. \\ Deuxième lieu - Msek (lieu-dit Sefri) : céréales, pains, levains, fours et amandes}

Sur lan linguistique, on constate la capacité de notre hôte qui avait séjourné dans

63 Msek est le lieu où A. Maghdad avait effectué son enquête à l'été 1992, et Sefri est l'un des points qu'elle avait visités (Figure 10). S., la mère aujourd'hui, était toute jeune mariée à l'époque. Les habitants de ce lieu-dit ont pour langue maternelle l'arabe marocain, contrairement à notre informatrice F. de Taounil; mais ce lieu a la particularité d'être en situation minoritaire parce qu'entouré de populations berbérophones. Les enfants en allant à l'école apprennent l'amazighe pour s'intégrer au groupe.

Figure 10 : Sur la route de Sefri - Msek février 2014 - au loin la Méditerranée et la ville de Bades

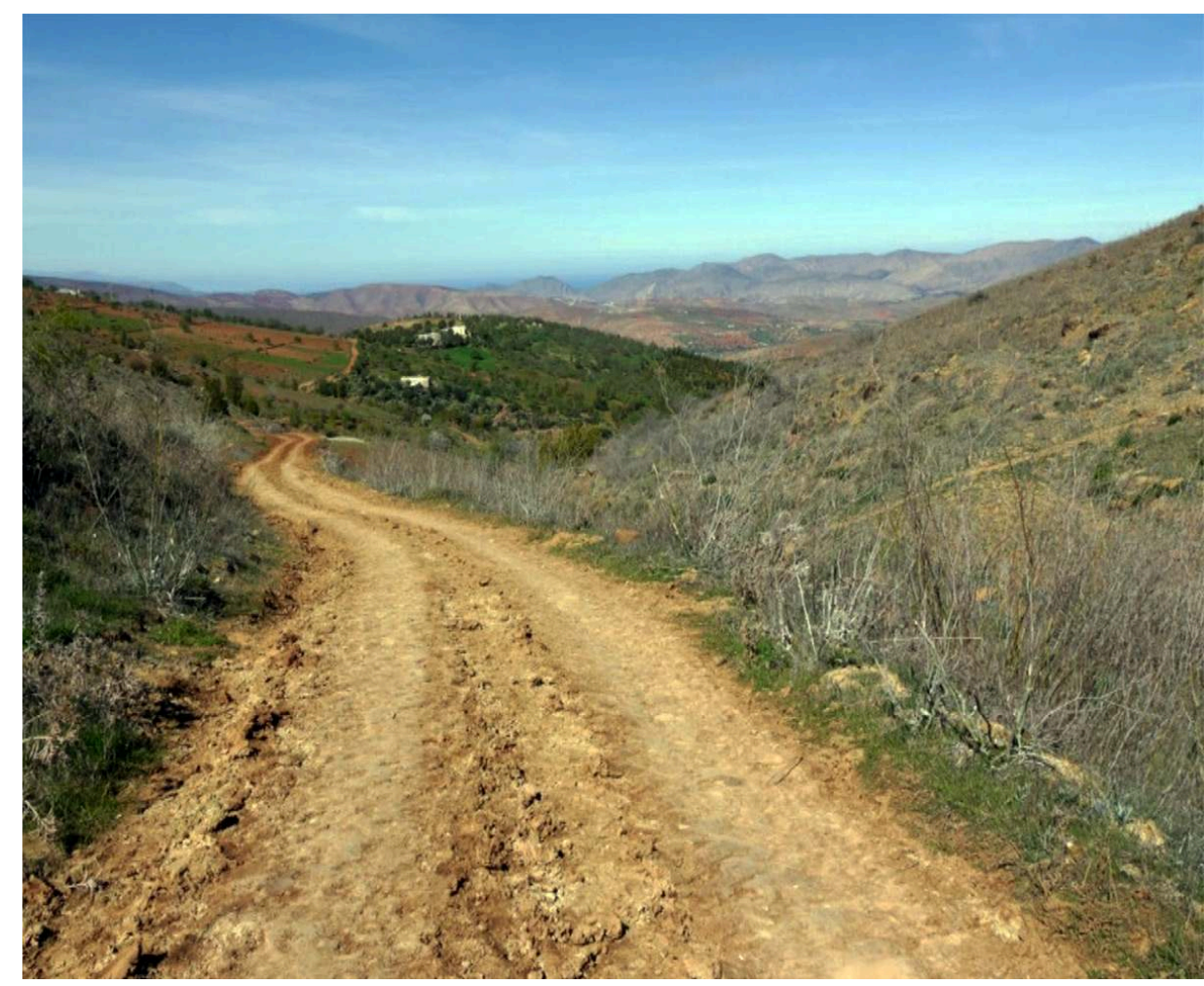

(C) D. Caubet

Bien qu'arabophones, nos informateurs ne se présentent pas comme appartenant aux Jbala, mais comme Rifains : nous avons donc affaire à des Rifains arabophones.

L'enquête ethnobotanique menée par Y. A.-Thomas a porté sur les céréales cultivées, leurs noms, ainsi que sur les techniques de fabrication du pain, et donc, sur le four et le combustible utilisé. 
Textes recueillis : enquête ethnobotanique, Y. A-Thomas, A. Maghdad, et D.

Caubet, Transcription D. Caubet

Le pain, le levain et les céréales (textes 7 à 11)

Amal : kiffăš katțayybi al-xobz dyālək ? (Fichier sonore 3)

(Texte 7) S. : kanțyybu lo xobz? kanžỉbu dïk-əl-qməh, kanəqqīw-hum, l-ḥ̌zor, u nəddīw-um lor-rha, nțəhnnu-hum f-r-rḥa, Bni Ḥdifa walla gìr hna... kanžïbu kanġorblu-hum 3əndna l-ġorbal

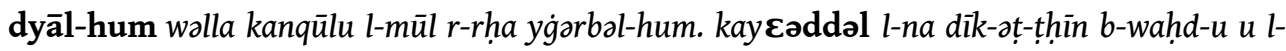
garšl-kbir b-wahdd-u u-s-ṣgìị bwahd-u

Amal : ol garš hūwa on-nuxxāla

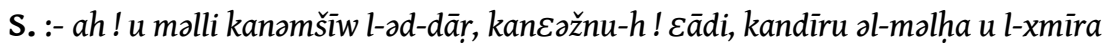

Amal : Comment fais-tu le pain?

Comment on fait le pain?

On prend le blé, on le nettoie - les petits cailloux- et on l'apporte au moulin, pour qu'ils le moulent, à Bni Hadifa, ou à coté... On le rapporte à la maison, on le tamise, on a un tamis spécial, ou bien on demande au meunier de le tamiser. Il nous met la farine d'un côté et le son épais à part et le son plus fin à part.

Ce média ne peut être affiché ici. Veuillez vous reporter à l'édition en ligne http:// journals.openedition.org/ethnoecologie/3070

Amal : garš c'est comme nuxxāla (son)?

Oui et quand on rentre à la maison, on le pétrit. C'est simple, on y ajoute du sel et du levain

Pour ce qui est du nom des céréales et des farines utilisées (Fichiers sonores 4 et 5), elle explique les types de céréales et la gestion des terres :

A. : š man thịin katdìr?

(Texte 8) S. : bhāal dāba, tḥīn d-əš-šEīr, d-əl-qmah

A. : katasmal l-xobz mxollat?

S. : bhāl āna fìya s-səkkār kayqūlu li nākūl zəema d-əš-šcìr, ḥna kanāklu d-əl-qməḥ, kanxallțu d-əl-qməh o șăfi !

D. : katšrīw al-qmah?

S. : la!

A. : l-ard dyāl-kum?

S. : dyāl-na! kayžỉbu-um l-na l-fallāha, kayhartu ol-aṛd dyāl-na kanəEțīw-ha l-um kayhartu kayEabbiww on-nașs dyā-um u l-blād dyāl-na kayacțīw-ha l-na.

ḥnāya, bba-h d-Moḥammed Eənd-u l-aṛd bazzāaf u kayəsți-ha l-ən-nās kayhartu-ha, kayaddīw l-ḥaqq dyā-hum kayžibu l-na haqq-na

A. : Quelle farine utilises-tu?

S. : Par exemple de la farine d'orge, de blé

A. : Tu fais du pain mélangé ?

S. : Par exemple, moi j'ai du diabète et ils me conseillent de prendre de l'orge, et donc ce qu'on mange, c'est du blé que l'on mélange, tout simplement !

D. : Vous achetez le blé ?

S. : Non!

A. : La terre est à vous?

S. : Oui, à nous! Ce sont les fermiers qui nous l'apportent, ils labourent notre terre. 
Parce que nous, le père de Mohammed, a beaucoup de terre et il la donne à des gens pour la cultiver et ils prennent leur part et nous apportent la nôtre; nous la leur donnons à travailler, ils prennent leur moitié et notre part, ils nous la donnent.

Mouliéras, dans la première partie du Maroc Inconnu, consacrée au Rif à la fin du XIX siècle (1895: 65), parle de l'importance de l'orge dans l'alimentation :

«Ils (les hommes) apportaient aux hôtes de la mosquée des poules, du poisson, du miel, du beurre, du pain d'orge (...) On ne connaît guère le pain de blé dans tout le Rif depuis Mthioua jusqu'à Nemours ${ }^{12}$, on en sème très peu, pour ne pas dire pas du tout. »

Ce média ne peut être affiché ici. Veuillez vous reporter à l'édition en ligne http:// journals.openedition.org/ethnoecologie/3070

Ce média ne peut être affiché ici. Veuillez vous reporter à l'édition en ligne http:// journals.openedition.org/ethnoecologie/3070

Nous abordons ensuite la fabrication du levain à Sefri (fichier sonore 6) :

Amal : l-xmira, kïfāš katEəddlu l-xmïra?

(Texte 9) - uh? hādị? hādāk fāš kanəعžnu, ḥna kanqūlu l-a l-xmīra d-əd-dāṛ (...)

lə-xmira, kanzuwwlu țərf mən əl-xobz, katEəddl-u f-wāhəd əž-žbāniyya, sțəl șgīwər wəlla ši hāža katšədd, təmmak u ndäfiw əl-ma u neəmlu-ha u nxəllțu-ha u nnəzzlu eli-ha ț-țhin u nġəttiw-ha katxmər

Le levain, comment tu le fais?

Hein ? Ça ? Quand on pétrit, nous on l'appelle le levain de la maison (...)

Le levain, on prélève un morceau de pain, tu le mets dans une bassine, un petit seau ou quelque chose qui ferme, et là on fait tiédir de l'eau, on l'ajoute, et on la mélange et on met de la farine dessus et on la couvre pour qu'elle lève.

Ce média ne peut être affiché ici. Veuillez vous reporter à l'édition en ligne http:// journals.openedition.org/ethnoecologie/3070

(Texte 10) u îla ma Eməlti-ha šay, Eməlti b-əl-xmīra d-əs-sūq $u$ șăfi, guwwuz nhar yūm$\bar{a} y \partial n, \dot{g} \partial d d a$ tșïb-u fsəd, șâfi, fi-ha dikk-əəə n-nəsma u dāk ši

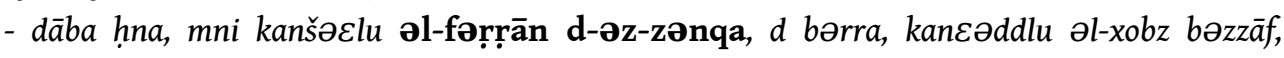

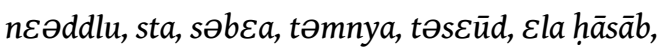

Et si tu ne l'utilises pas (la levure faite maison) et que tu mets seulement de la levure du marché, tu laisses passer un jour ou deux et tu t'apercevras qu'il est moisi (le pain), avec de la moisissure, et tout ça...

- Par exemple, nous, quand on utilise le four extérieur (litt. 'de la rue', voir plus bas), de dehors, on prépare le pain en quantité, on en fait $6,7,8$, 9, ça dépend. (Fichier sonore 7)

Ce média ne peut être affiché ici. Veuillez vous reporter à l'édition en ligne http:// journals.openedition.org/ethnoecologie/3070

À ce propos Mouliéras (1899 : 279) dit qu'à la fin du XIXe siècle, chez les Ghomara (R'mara selon son orthographe) : «on se contente de faire le pain une fois par mois ». 
-ah kanعaddlu šwiyya, māši bazzāf, māši šwiyya

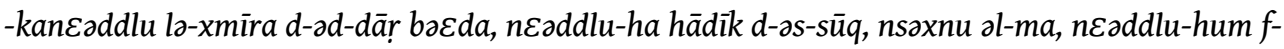

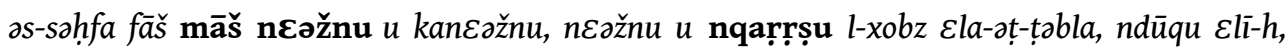
nxalliw-oh yxmor

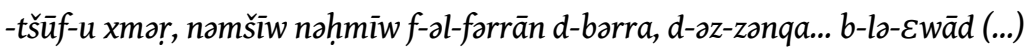

-ehhe! kayțib u kancaddlu fi-ha al-xobz u nraddu Eli-h yțīb u nzawwlu-h

u sāâi!

Le levain ? Est-ce qu'on en met beaucoup ou peu?

Oui, on en met une petite quantité, ni trop ni pas assez.

Par contre, on utilise du levain fait à la maison, on prend du levain du marché (qu'on achète), on fait chauffer l'eau dans le récipient dans lequel on pétrit, et on pétrit; on pétrit puis on forme les pains sur la table, on appuie bien, et on le laisse lever.

Quand tu vois qu'il a levé, on va faire chauffer le four de dehors, avec des morceaux de bois.

Oui, quand il est prêt on y met le pain, on le laisse cuire et puis on l'enlève, et voilà !

Ce média ne peut être affiché ici. Veuillez vous reporter à l'édition en ligne http:// journals.openedition.org/ethnoecologie/3070

\section{Msek : Classification des amandes}

Le fils de notre informatrice, Y. 13 ans, questionné par Y. Aumeeruddy-Thomas sur les types d'amandes présentes chez eux en a cité trois types (Fichier sonore 9).

76 (Texte 12) waḥd-ən-nūe, kayatharras gìr b-al-fumm, walla b-ol-ïdd-āyən

\section{(...)}

hāda ma-hūwa kaythorras b-əl-īdd, ma hu... dəg்ya kaytharras b-la-hžor. Kāyn wāhad axōọ, șeīb

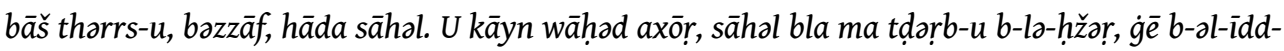
àyon

Il y a une sorte qu'on peut casser avec la bouche, ou bien à la main (...) mais celle-ci ne se casse pas à la main, elle... elle se casse facilement avec un caillou. Il y en a une autre sorte qui est très difficile à casser, alors que celle-ci est facile. Et enfin, il $y$ en a une qui est facile, pas besoin de la frapper avec une pierre, juste à la main.

Ce média ne peut être affiché ici. Veuillez vous reporter à l'édition en ligne http:// journals.openedition.org/ethnoecologie/3070

77 Il donne son nom, qui, à notre connaissance, n'a jamais été répertorié avant notre mission, un mot hybride combinant arabe et berbère (Fichier sonore 10) ${ }^{13}$ :

(Texte 12) hādāk lli kaythərrəs dəg்ya, kayqūlu l-u 'bu-ġummās-i'... bu-gummās-i!

Celle qui se casse facilement, ils l'appellent bu-gummāasi... bu-gummāasi! (Voir plus bas l'explication de ce nom)

Ce média ne peut être affiché ici. Veuillez vous reporter à l'édition en ligne http:// journals.openedition.org/ethnoecologie/3070 


\section{Msek : Analyse linguistique à partir des textes 7 à 12}

\section{finale.}

(Texte 7) kanțyybu ol-xobz? 'Comment on fait cuire le pain? ent aux déclaratives), et si on a bien une montée sur le terme principal (xobz), il n'y a par contre, ni remontée finale (question ?) ni baisse finale généralement associée aux déclaratives.

(Texte 10) l-xmira? bazzāf walla šwiyya zaEma? Le levain ? Si on en met beaucoup ou pas ?

\section{Pour ce qui est de la morphosyntaxe}

87 Le préverbe d'inaccompli est systématiquement ka-, comme à Taounil et contrairement à beaucoup de parlers jbala ${ }^{15}$ : kanžïbu dikk-əl-qməh, kanəqqīw-hum (accord au pluriel, voir point suivant) 'on apporte ce blé, on le nettoie'.

88 - Sous l'influence du berbère on remarque un accord au pluriel de mots ailleurs singuliers al-qmah 'le blé' (exemple ci-dessus), tout comme pour l'eau, al-ma, dont l'accord varie entre le masculin singulier et le pluriel :

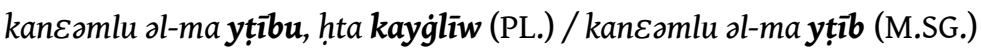

'Nous mettons l'eau à chauffer jusqu'à ce qu'elle bout / Nous mettons l'eau à chauffer'.

89 - Une particule de futur māš que l'on trouve dans de nombreux parlers préhilaliens,

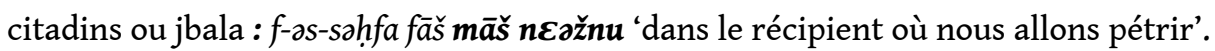




\section{Nous avons relevé deux innovations syntaxiques} parlers très éloignés géographiquement, puisqu'il s'agit de Jijel (Est Algérien), mais très proches dans leur histoire, puisqu'ils font tous les deux partie de parlers 'villageois', selon la terminologie de William Marçais (voir Marçais et Guîga 1925- Avant-propos : XXVIII-XXXVII).

91 Il s'agit de l'emprunt du présentatif berbère $\underline{d}$ (réalisé $[\mathrm{d}]$ ou [d] en arabe), très rarement (ou jamais ?) signalé dans des parlers arabes au Maroc, et qui est très courant à Jijel (Marçais 1956 t. II : 462-466). Il avait été signalé par Maghdad (1993:62), dont le mémoire resté inédit est repris dans la synthèse de Caubet (2017:121). Maghdad l'avait recueilli, il y a vingt-cinq ans, chez un vieil homme qui avait 20 ans au moment de la guerre du Rif (1921-1926) : « Texte X : kan bababa d-el-qüyed 'mon père était le caïd' » Nous l'avons retrouvée en 2014 chez notre informatrice dans un texte où elle décrit les farines utilisées pour le pain :

ḥna kanāklu d-əl-qməh, kanxəlltu d-əl-qməḥ o șäfi !

Ce que nous mangeons, c'est du blé; c'est avec du blé que nous le mélangeons (le seigle), et voilà !

\section{par le pronom affixe et la particule possessive arabe $d$ :}

hnnāya, bba-h d Moḥaməd Eənd-u l-aṛ bəzzā $f$

Nous, le père de Mohamed a beaucoup de terre.

93 Kossman (2017) l'analyse comme une construction inspirée du berbère, citant le cas du parler de Jijel (Algérie) décrit par Ph. Marçais :

" On the level of the noun phrase, some Maghrebian Arabic varieties have a Berberinspired construction in making possessor phrases to kinship terms. In Berber, most kinship terms are inherently possessed, and a possessor phrase is only admitted in combination with a possessive pronoun, for example $g^{w} m a-s$ 'his/her brother'; $g^{w} m a-s n$ Kltum 'Keltouma's sister,' lit. 'her sister of Keltouma' (Tashelhiyt/ B|MA; Galand, 2010, p. 139). In a number of Maghrebian Arabic varieties, this construction has been taken over, and one finds phrases such as $x t-u$ 'his sister,' $x t-u$ ddə mhəmməd 'Mohammed's sister,' lit. 'his sister of Mohammed' (Jijel/A|AL; Marçais 1956: 611, for discussion of similar constructions in some Moroccan varieties, see Fischer 1907, Heath 2002 : 463-464). »

\section{Pour ce qui est du lexique}

On a des termes empruntés au berbère, comme ibāwen 'fèves', žru 'chiot' ; et des termes

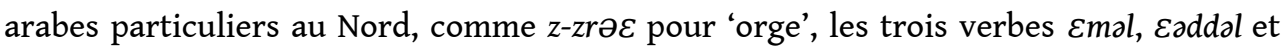
dār (le verbe du Maroc central) pour dire 'faire, mettre', marquant un changement linguistique en marche.

Et enfin le nom de l'amande bu-gummāsi. Ce nom hybride est formé à partir d'une racine pan-berbère $\sqrt{ } \gamma M S$ (voir en berbère tuymest, tiłmest 'dent, molaire', pluriel, tuymas, tiymasen), combinée à un schème nominal arabe marocain: bu (père) + un attribut (voir Caubet 1993, vol 2:295). On citera bu kařs, bu nif 'le type au gros ventre, au gros nez', bu l-lohya 'le barbu'. Bu agit comme un localisateur auquel on assigne des qualités ou des défauts. 

de la racine berbère, $\sqrt{ } \mathrm{YMS}$, d'un schème arabe intensif avec gémination de la consonne médiane > gummās, auquel est ajouté le suffixe adjectivant (nisba) $-i>\dot{g} u m m \bar{a} s-i>b u-$ gummāss- $i$ : « celui que l'on peut casser avec les dents ».

Tableau 3 : Traits phonétiques

\section{Caubet}

Tableau 4 : Quelques points de morphosyntaxe

\begin{tabular}{|c|c|}
\hline & Msek \\
\hline Diphtongue aw et ay & əl-lawz 'amandes', z-zaytūn 'les oliviers' \\
\hline 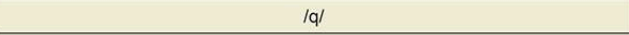 & réalisé [q] \\
\hline$/ d /$ réalisé $[d]$ & hāyda, hādi \\
\hline $\begin{array}{l}\text { amuïssement du } / \mathrm{h} / \text { dans les pronoms personnels de 3ème personne, mais } \\
\text { pas dans tous les cas }\end{array}$ & 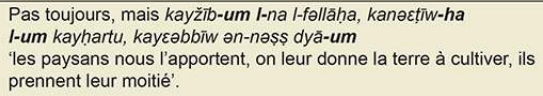 \\
\hline
\end{tabular}

D. Caubet

\section{Msek : Analyse ethnobotanique}

\section{Le pain, le levain et les noms des céréales}

Le pain préparé à partir de blé ou d'orge (qməh ou zrəc) qui sont co-plantés avec des amandiers, arbre dominant de cette partie du Rif. Le pain est toujours marqué de traces, celles de notre hôte étant une série de trous, qui non seulement ont pour but de faciliter la cuisson du pain, mais représente aussi la marque personnelle de chaque maisonnée. Ceci laisse supposer qu'à une époque antérieure, plusieurs femmes pouvaient à certains moments utiliser le four d'une maison voisine ou de quelqu'un de la famille, ces ornementations servant alors à ne pas mélanger les pains.

Le jour où nous avons visité Msek (février 2014), il ne restait au village que 3 familles, suite à un exode rural très important et nous avons pu constater la grande solitude de notre hôte.

Comme le montre les données de l'enquête, le pain communément fabriqué à la maison est obtenu à partir de farine d'orge ou de blé récolté sur leurs terres, mais généralement travaillé par des fermiers pour eux contre une rétribution en nature (Figure 11). La farine est obtenue en apportant les céréales au moulin, donc chez un spécialiste qui peut le tamiser ou non. Un plat à pétrir nommé şộfa est utilisé pour pétrir la farine avec du sel, de l'eau et du levain. C'est un grand plat en terre cuite, aussi appelé tažafnit (Caubet 2016: 172). Ce pain est caractérisé par l'utilisation de levain fabriqué à la maison par opposition au levain chimique que l'on peut acheter au souk. Comme dit notre hôte : Le levain l-xmira, obtenu localement est fabriqué à partir d'un mélange équilibré de morceau de pâte à pain, d'eau et de sel, que l'on place à l'abri et 
qu'on ajoute au pain afin qu'il lève. Ce levain confère une grande tenue au pain qui de ce fait se conserve plus longtemps. Notre informatrice n'utilise que des levains faits à la maison, mais elle n'utilise pas le terme de bəldi ou romi ; elle oppose l-xmira $d$-əd-dār (le levain de la maison) à əl-xmira d-əs-sūq (le levain du marché). Une fois de plus et comme constaté plus haut, bien qu'il ne s'agisse pas d'une opposition binaire entre baldi et romi, le levain et le pain fait-maison sont fortement valorisés par rapport au levain du souk qui donne un mauvais pain qui s'abime très vite (Textes 10 et 11) ci-dessus).

Figure 11 : Pain fabriqué par $\mathrm{S}$. à Msek avec les traces (cette série de trous) qui correspondent à sa marque de fabrique

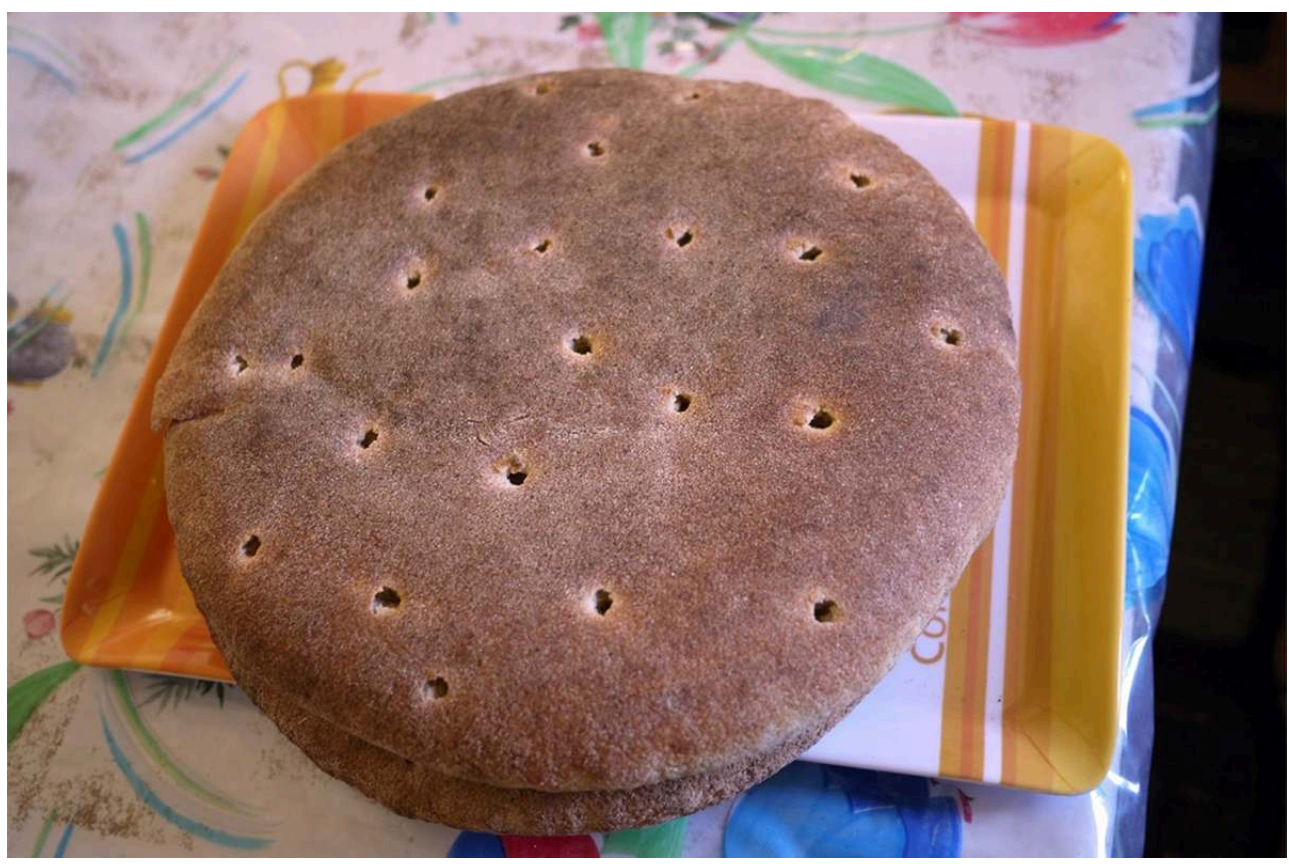

(c) D. Caubet

101 Notre hôte nous invita ce jour-là à partager son pain avec un plat de lūbya, haricot blanc en sauce. Ainsi, là comme partout ailleurs dans le Rif, le pain accompagné de divers plats de fève, haricot, purées diverses fabriquées à base de lentilles, pois chiche, fèves, le tout agrémenté d'huile d'olive, constituent des éléments de base de la cuisine rifaine et jbala.

102 L'orge se dit zroE (terme qui désigne le blé dur ailleurs dans le pays, où l'orge se dit šEir), et le blé dur, qmoh. Durant notre échange S. utilise d'ailleurs plusieurs fois le terme šsir pour l'orge, contrairement à son habitude, terme qu'elle connaît parfaitement et qu'elle utilise du fait de notre présence. (Texte 7 ci-dessus).

\section{Msek : Le four - La zonqa (rue) dans le monde rural}

103 Notre informatrice désigne le four où l'on fait cuire le pain comme əl-fərrān d-əz-zənqa, d-bərrra 'le four extérieur, de dehors' ; or ce terme désigne la rue en milieu urbain et son emploi à la campagne est inédit. (Texte 11).

Tchirine Mekideche (1996) dans son travail sur la zənqa en milieu citadin écrit :

«La zanka représente ainsi un espace écran, tampon, intermédiaire entre l'espace intérieur réservé à la femme et l'espace extérieur réservé à l'homme ». 
La zənqa apparaît donc en ville comme un espace d'autonomisation et de socialisation, un espace intermédiaire, de passage entre la dār (maison) et l'espace public, masculin. La zənqa est vue positivement, comme protégeant l'espace privé de l'extérieur. Elle poursuit :

«Concrètement, architecturalement, la zanka est tout espace centré sur le domicile et à la libre disposition des enfants. "

Dans les autres régions du Rif et Jbala que nous avons visitées, le four est couramment appelé forna, même si le four principal de la ville de Chefchaouen est appelé farrān, terme que l'on retrouve ailleurs chez les Jbala. Il s'agit en effet d'un grand four public où chacun apporte son pain pour la cuisson et qui est surveillé par un homme, mūl lforṛān, le maître du forṛān.

07 À Sefri, la zənqa désigne un espace intermédiaire, accessible aux femmes, puisque ce sont elles qui construisent, utilisent et entretiennent le four. Il est assez haut $(1 \mathrm{~m} 80$ voir Figure 12), et son ouverture est située à plus d'un mètre du sol, contrairement à beaucoup d'autres régions où le four est bas et l'ouverture au ras du sol.

Il partage avec la maison un espace privé, protégé par des clôtures et séparé du vrai extérieur, espace public par des figuiers de Barbarie et des épineux.

Figure 12 : əl-fərrān d-əz-zənqa, Msek 2014

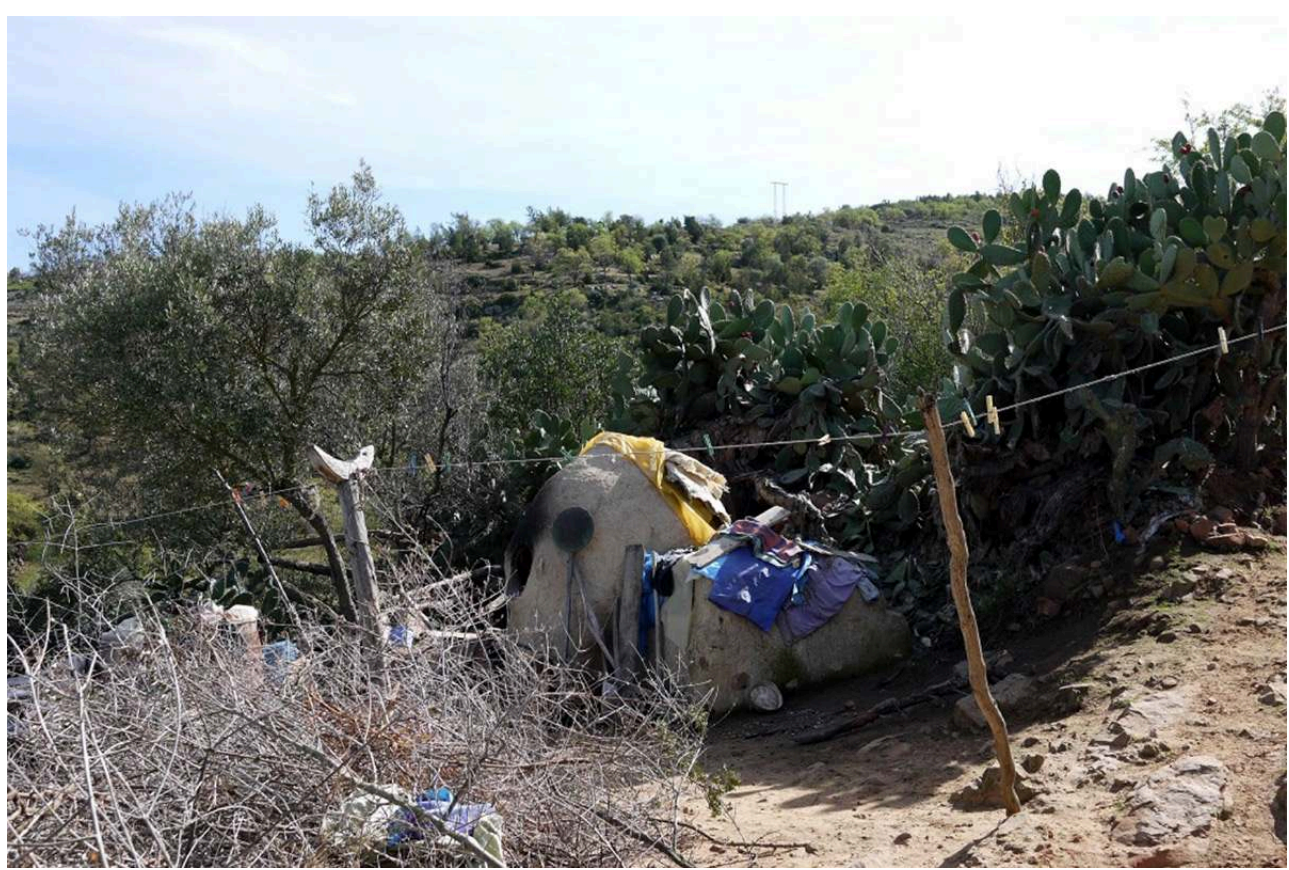

(c) D. Caubet

\section{Msek : Four et amandes}

Les amandes à coques dures (dites $l-l \bar{u} z \varepsilon \bar{a} d i$, les amandes ordinaires) les plus répandues dans cette région, s'opposent aux amandes à coques tendres qui peuvent s'ouvrir avec les dents, dites bu-gummāsi (voir les termes snān et ghemmas à Bni Boufrah, Delplancke et Aumeeruddy-Thomas dans ce volume). Ce sont les amandes greffées (par talqìm), variétés venues d'ailleurs qui ont des coques tendres (Texte 12 ci-dessus). 
110 La nature de la coque des amandes fonde ici un des schèmes classificatoires binaires «coques dures qui se cassent avec des pierres" et "coques tendres plus ou moins faciles à casser à la main ou avec la dent » et renvoient à l'opposition dans l'ensemble de l'agroécosystème à amandier à deux façons de gérer les amandiers, la façon locale qui donne des l-lūz baldi à coques dures issus de semis naturels ou spontanés et le greffage qui a été apporté par les espagnols qui donne des l-lūz romi à coques tendres.

111 Un lien intéressant est à établir entre les amandes et le four, puisque les coquilles des amandes à coques dures servent de combustible dans le four (Figure 13). Ces coques amandes, une fois brûlées sont jetées dans un lieu précis en face de la maison. Il est possible que ces sols noirs très riches en charbon qui en résultent puissent être d'un usage important pour enrichir les terres agricoles, mais nous n'avons pas pu le vérifier.

Figure 13 : Le four de la zənqa et l'usage des coques d'amandes à coques dures pour allumer le feu, Msek, 2014

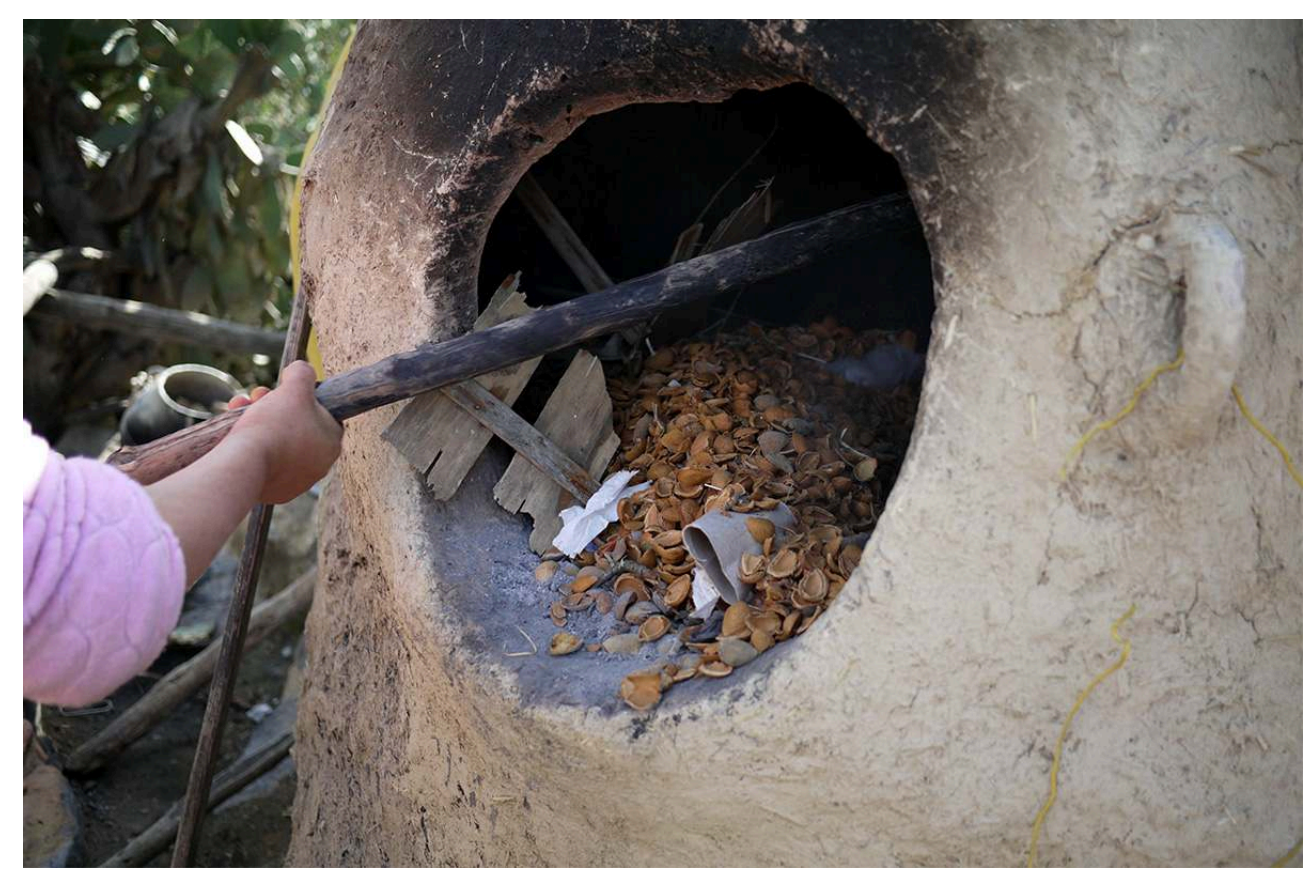

(c) D. Caubet

112 Deux instruments sont utilisés l'un pour enfourner le pain; il s'agit d'une grande pelle appelée matrah (Figure 14) qui ressemble à une grande rame, et pour nettoyer les centres, un balai se terminant par des lanières de tissus appelé akennas (Figure 15). 
Figure 14 : Metrah, instrument utilisé pour placer le pain dans le four

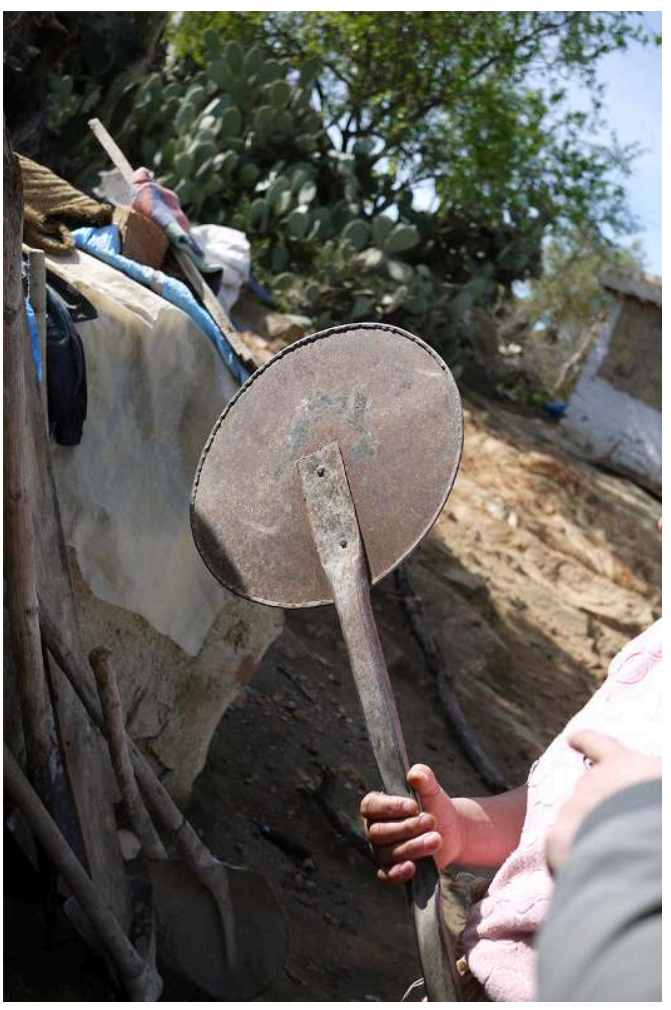

(c) D. Caubet

Figure 15 : Akennas : instrument utilisé pour nettoyer le four

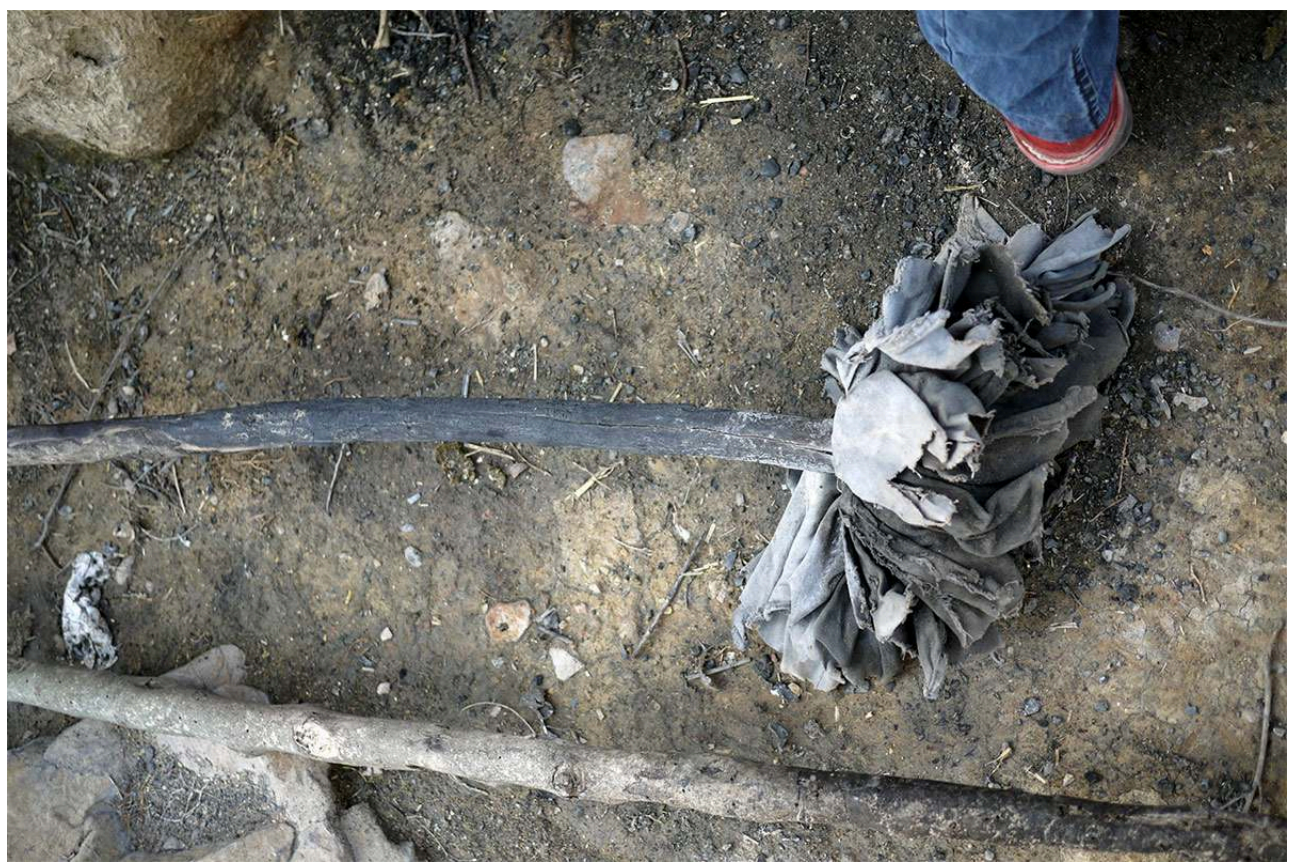

(c) D. Caubet

113 Le four comme dans toutes les autres régions du Rif est fabriqué localement par les femmes avec de l'argile (trāb) et de la balle de céréales (tban). Nous avons observé des fours individuels de ce type, avec des variantes au niveau de la forme dans toutes les 
régions jbala que nous avons cité précédemment, à savoir, chez les Bni Ahmed, à Ouezzane, Ain Mediouna etc. Dans ces régions, des femmes spécialisées dans la fabrication de ces fours sont parfois mises à contribution. Sont rajoutées à l'argile et la balle de céréales qui constitue le mortier, d'anciennes poteries (des morceaux de vieux plats à tajine) pour le fortifier et augmenter ainsi sa capacité de résister à la chaleur.

\section{Conclusion}

Les différentes missions conjointes que nous avons effectuées (2013-2014) ont été extrêmement enrichissantes. Les données qui ont pu être recueillies, l'ont été grâce à la combinaison des techniques d'enquête propres à chaque discipline, et au fil des missions, nous avons réussi à mettre au point une technique mixte qui permettait au linguiste d'enregistrer de façon aussi précise que possible et à l'ethnobotaniste d'avoir accès à une traduction permettant de suivre le déroulé de l'entretien afin de pouvoir poursuivre avec des questions pertinentes qui concerne le champ de l'ethnobotanique.

115 Les terrains où nous avons travaillé, proches de la ville d'el Hoceima, sont aujourd'hui au cœur de l'actualité et ne seraient pas forcément accessibles pour nous aujourd'hui. Les données sont d'autant plus précieuses.

116 Tant que le mémoire de A. Maghdad (1993) n'avait pas été publié ${ }^{16}$, il n'existait pas de description de ces parlers situés dans des zones frontalières et inédites - quand par exemple, la darija se trouve en position de langue minoritaire, ce qui est rare.

117 L'ethnobotanique de terrain, s'appuie toujours sur les catégories locales qui s'expriment par la langue, et la méthode consiste à relever les termes, la nomenclature et les taxinomies locales. Les travaux développés dans le Rif, tels que ceux initiés sur la diversité variétale des figuiers, ou les techniques autour de l'olivier (Hmimsa et al. 2012, Aumeeruddy-Thomas et al. 2017) illustrent cette démarche. La rencontre avec la linguistique de terrain nous a permis d'interroger des situations de frontières linguistiques; mais les données ethnobotaniques montrent une absence de frontière sur les savoirs et savoir-faire concernant les céréales, le pain, le four et un ensemble plus vaste de savoirs sur la nature (usage du bois de Thuya, des plantes adventices, classification des amandes etc.) même si les techniques interrogées dans les deux sites ne sont pas exactement les mêmes. La confrontation à des données récoltées dans d'autres sites, notamment chez les Jbala, montre des variations et en même temps des savoirs et savoir-faire partagés et une grande unité entre le Rif plutôt berbérophone, mais néanmoins avec des groupes arabophones comme nous l'avons vu à Msek (au sens des cartes de Renisio et Maurer) et les Jbala arabophones.

118 À Taounil, la situation de notre informatrice ayant vécu plusieurs années à Ceuta, et revenant ensuite dans son village dénote une relative solitude car très différente des autres femmes du village. Amazigh de naissance, elle utilise néanmoins couramment l'arabe pour expliquer la fabrication de zambu, la cueillette des asperges, ou l'identification des plantes sauvages, même si elle donne aussi au fil de l'entretien quelques équivalents en berbère. Au-delà de la langue, sa gestuelle, une autre forme de langage, autour de la fabrication de zombu, de la cueillette des asperges, lors de la visite des jardins, démontre que c'est au contact du terroir qu'émergent les lexiques complexes de ces mondes ruraux, mais aussi les gestes au cours d'un cheminement dans des lieux connus, lui permettant de nous montrer ce qui fait sens : l'ancienne 
maison en bois en عarcar (Thuya et cyprès), aujourd'hui abandonnée, les limites (hhūud) des parcelles à peine visibles, les anciens réservoirs de céréales enfouis sous la terre (matmōra) et toutes ces herbes sauvages (baqqōla) qui sont baldi et sont qualifiés par opposition à un ailleurs (romi) toujours moins bon, plus piquant. De même, à Msek, c'est à travers la manipulation active de son four que $\mathrm{S}$ nous explique ce que faire cuire le pain dans son propre four (farrāan $d$-əz-zənqa) signifie, outre toute les explications données sur la qualité des pains, du levain fait-maison et l'importance que recouvre tout son univers familial qui s'oppose au souk et à la ville où elle doit quand même se rendre pour des soins à l'hôpital dans des conditions souvent très difficiles. Son univers familier intègre, outre les membres de sa famille, les jardins, avec l'omniprésence de l'amandier, les céréales diverses, les fèves et tous les animaux domestiques, chiens, poules, moutons, une communauté de vie que nous n'avons pas évoquée dans ce papier. Malgré la solitude prégnante de ces deux femmes, dans un monde en forte transition, leur attachement à leur maison et aux lieux, la très grande qualité des techniques alimentaires qu'elles continuent de perpétuer, nous a semblé être la marque d'une forte identité culturelle, signe d'une résilience extraordinaire malgré des vies extrêmement difficiles.

\section{BIBLIOGRAPHIE}

Abou El Haja H. 1995 - Habla árabe en duwar. Srema (tribu Bni Qorra, provincia de Taunat). Textos (transcripción, traducción y notas) y estudio fonético y morfológico. Mémoire de Licence d'Espagnol sous la direction de Simon Lévy. Rabat, Université Mohamed V, 95 p.

Arsenne J. 2016 - Preliminary results on the Arabic spoken in Jnanate, Northern Morocco. In : Grigore G. \& BiȚună G. (Ed.) Arabic Varieties: Far and Wide. Proceedings of the $11^{\text {th }}$ International Conference of AIDA, Bucharest 2015. Bucharest, Editura UniversităȚii din București : 73-78.

Aubaile-Sallenave F. 1999 - Les nourritures de la femme accouchée dans le monde arabomusulman. Médiévales 33, Numéro Spécial : Cultures et nourritures de l'occident musulman: essais dédiés à Bernard Rosenberg : 103-124.

Aumeeruddy-Thomas Y., Hmimsa Y., Ater M. \& Khadari B. 2014 - Beyond the divide between wild and domesticated: spatiality, domesticity and practices pertaining to fig (Ficus carica L.) and olive (Olea europaea L.) agroecosystems in Morocco. In : Chevalier A., Marinova E. \& Peña-Chocarro L. (Ed.) Crops and people: choices and diversity through time. Brussels, Earth EU, London, OXFAM : 191-197.

Aumeeruddy-Thomas Y., Clochey L., El-Ghazaz F. \& Caubet D. 2014 - Céréales et huiles d'olive : savoirs, savoir-faire techniques et relations d'échanges entre deux groupes sociaux voisins, Jbala (Ain Mediouna) et Hyayna (Switeyyin) du Nord du Maroc. Communication au Congrès International Lenguas, Memorias y Lecturas de la Alteridad: Jbala, Ghomara y Rif (Norte de Marruecos), 8-9-10 Sept 2014, Saragosse.

Aumeeruddy-Thomas Y., Moukhli A., Haouane H. \& Khadari B. 2017 - Ongoing domestication and diversification in grafted olive-oleaster agroecosystems in Northern Morocco. Regional 
Environmental Change 17 (5) : 1315-1328. [En ligne] DOI 10.1007/s10113-017-1143-3._https:// www.academia.edu/32587199/

Ongoing_domestication_and_diversification_in_grafted_olive_oleaster_agroecosystems_in_Northern_Morocco. Consulté le 15 juillet 2017.

Behnstedt P. 2002 - La frontera entre el Bereber y el Árabe en el Rif. Estudios de dialectología norteafricana y andalusí $6: 7-18$.

Behnstedt P. 2005 - Materialien für einen Dialekatlas von Nordost-Marokko I: Tiernamen, Teil 1: Einleitung, Transkription, Ortnamenverzeichnis, Karten. Estudios de dialectología norteafricana y andalusí 9 : 7-72.

Behnstedt P. 2007 - Materialien für einen Dialekatlas von Nordost-Marokko I: Tiernamen, Teil 2: Kommentare, Bibliographie. II: Mensch: Körperteile, Teil 1: Karten, Teil 2: Kommentare. Estudios de dialectología norteafricana y andalusí 11 : 7-57.

Behnstedt P. \& Benabbou M. 2002 - Zu den arabischen Dialekten der Gegend von Tāza (Nordmarokko). In : Arnold W. \& Bobzin H. (Ed.) Sprich doch mit deinen Knechten aramäisch, wir verstehen es!. 60 Beiträge zur Semitistik Festschrift für Otto Jastrow zum 60. Geburstag,. Wiesbaden, Harrassowitz Verlag : 53-72.

Cadi K. 1987 - Système verbal rifain : forme et sens linguistique tamaziqht (nord marocain). Paris, SELAF.

Caubet D. 2017 - Les parlers du Nord-Ouest marocain à partir de corpus recueillis dans la région en 1992-1995 (sous la direction de Simon Lévy). In : Vicente Á., Caubet D. \& Naciri Azzouz A. (Ed.) La région du Nord-Ouest marocain: Parlers et pratiques sociales et culturelles. Zaragoza, Prensas de la Universidad de Zaragoza : 109-142.

Caubet D. 2016 - The dialect of Msek - Beni Itteft (Al Hoceima), on the borders with Berber. In : Grigore G. \& BiȚună G. (Ed.) Arabic Varieties: Far and Wide. Proceedings of the 11th International Conference of AIDA, Bucharest (2015). Bucharest, Editura UniversităȚii din București : 163-173.

Caubet D. 1993 - L'arabe marocain, tomes I (Phonologie et morphosyntaxe) et II (Syntaxe et catégories grammaticales, textes) Louvain-Paris, Peeters, 273 p. et 402 p.

Colin G.S. 1921 - Notes sur le parler arabe du nord de la région de Taza. Bulletin de l'Institut Français d'Archéologie Orientale $18: 33-119$.

El Jattari K. 2017 - Contact de parlers arabe et berbère en zone du Rif : Région de Bni Itteft. In : Vicente Á., Caubet D. \& Naciri Azzouz A. (Ed.), La région du Nord-Ouest marocain: Parlers et pratiques sociales et culturelles. Zaragoza, Prensas de la Universidad de Zaragoza : 315-326.

Fischer A. 1907 - Eine interessante algerisch-marokkanische Genetivumschreibung. Zeitschrift der Deutschen Morgenländischen Gesellschaft 61 :178-185.

Franconie H., Chastanet M. \& Sigaut F. 2010 - Couscous, Boulgour et Polenta. Transformer et consommer les céréales dans le monde. Paris, Karthala, $477 \mathrm{p}$.

Heath J. 2002 - Jewish and Muslim dialects of Moroccan Arabic. London, New York, Routledge Curzon, $598 \mathrm{p}$.

Hmimsa Y. \& Ater M. 2008 - Agrodiversity in the traditional agrosystems of the Rif mountains (North of Morocco). Biodiversity: Journal of Life on Earth 9 (1 \& 2) : 78-81.

Hmimsa Y. \& Ater M. 2010 - Le savoir-faire paysan : quelques exemples. Regards sur les patrimoines et les terroirs des Jbala. 3ème Forum International Planète-Terroirs. Chefchaouen-Maroc, Ministère de la culture : 73-78. 
Hmimsa Y, Aumeeruddy-Thomas Y. \& Ater M. 2012 - Vernacular Taxonomy, Classification and Varietal Diversity of fig (Ficus carica L.) Among Jbala cultivators in Northern Morocco. Human Ecology 40 : 301-313. [En ligne] DOI 10.1007/s10745-012-9471-x. URL : https://www.academia.edu/ 32143756/

Vernacular_Taxonomy_Classification_and_Varietal_Diversity_of_fig_Ficus_carica_L._Among_Jbala_cultivators_in_Northern_Morocco [Consulté le 15 juillet 2017].

Hminsa Y., Aumeeruddy-Thomas Y. \& Ater M. 2017 - Lexique sur les figuiers: exemple des variétés nommées chez les Beni Ahmed (Jbala, Rif occidental). In : Vicente Á, Caubet D. \& NaciriAzzouz A. (Ed). La région du Nord-Ouest marocain : Parlers et pratiques sociales et culturelles. Saragosse, Prensas de la Universidad de Zaragoza : 265-274

Iraqui-Sinaceur Z. (Ed.). 1993 - Le Dictionnaire COLIN d'Arabe Dialectal Marocain. Rabat, Éditions El Manahil, (8 volumes), $2107 \mathrm{p}$.

Khoukh A. 1993 - El habla jebli de la ciudad de Wazzan en 1992. Mémoire de licence d'espagnol non publié sous la direction de Simon Lévy. Rabat, Université Mohamed V, 70 p.

Kossman M. 2017 - Berber-Arabic Contact. In : Oxford Research Encyclopedia of Linguistics. [En ligne] URL : http://linguistics.oxfordre.com/view/10.1093/acrefore/9780199384655.001.0001/ acrefore-9780199384655-e-232?rskey=hOxCDP\&result=1 [Consulté le 21 juillet 2017].

Lafkioui M. 2007 - Atlas linguistique des variétés berbères du Rif, (extracts), Berber Studies 16. Köln, Rüdiger Köppe Verlag. [En ligne] URL : http://menalafkioui.weebly.com/uploads/ 7/2/9/5/7295665/lafkioui_extract_atlas_2007.pdf [Consulté le 15 juin 2017].

Lévi-Provençal E. 1922 - Textes arabes de l'Ouargha, dialecte des Jbala (Maroc septentrional). Paris, E. Leroux, $285 \mathrm{p}$.

Maghdad A. 1993 - El habla árabe en el Aduar de Msek (Textos, Traducciones, Notas y Compendio). Mémoire de licence d'espagnol non publié sous la direction de Simon Lévy, Rabat, Université Mohamed V, $88 \mathrm{p}$.

Marçais Ph. 1956 - Le parler arabe de Djidjelli. Paris, Maisonneuve, 648 p.

Marçais W. \& Guîga A. 1925 - Textes arabes de Takroûna (Textes, Transcription et Traduction annotée) Tome I. Paris, Imprimerie Nationale, 426 p.

Maurer G. 1968 - Les paysans du haut Rif central. Revue de Géographie du Maroc 14 : 3-70.

Mekideche T. 1996 - La « zanka » : espace d'autonomisation et de socialisation dans la ville au Maghreb. In : Herbaut C. \& Wallet J.-W. (Ed.) Des sociétés, des enfants. Le regard sur l'enfant dans diverses cultures. Paris, Éditions la Licorne/L'Harmattan : 49-60. [En ligne] URL : https://enfancebuissonniere.poivron.org/

La_\%22zanka\%22_\%3A_espace_d\%27autonomisation_et_de_socialisation_de_l\%27enfant_dans_la_ville_au_Maghreb [Consulté le 9 juin 2017].

Mercier H. 1951 - Dictionnaire Arabe-Français. 2ème éd. Rabat, Les Éditions de la Porte, 290 p.

Monkachi M. 1997 - L'alimentation traditionnelle dans les campagnes du nord du Maroc. Médiévales 33, Numéro Spécial : Cultures et nourritures de l'occident musulman: essais dédiés à Bernard Rosenberg : 91-102. [En ligne] URL : www.persee.fr/doc/medi_0751-2708_1997_num_16_33_1397 [Consulté le 23 juillet 2017].

Morales J., Pérez Jordá G., Peña-Chocarro L., Bokbot Y., Vera J.C., Martinez Sánchez R.M. \& Linstädter J. 2016 - The introduction of South-Western Asian domesticated plants in NorthWestern Africa: An archaeobotanical contribution from Neolithic Morocco. Quaternary 
International 412 : 96-109. [En ligne] URL : http://dx.doi.org/10.1016/j.quaint.2016.01.066

[Consulté le 15 juin 2017].

Moscoso García F. 2003 - El dialecto árabe de Chauen (Norte de Marruecos). Cádiz, Universidad de Cádiz, 382 p. (Estudio lingüístico y textos).

Mouliéras A. 1895 - Le Maroc inconnu - 1ère partie : exploration du Rif(Maroc Septentrional). Paris, [à compte d'auteur], $221 \mathrm{p}$.

Mouliéras A. 1899 - Le Maroc inconnu - $2^{\text {ème }}$ partie : exploration du Djebala (Maroc Septentrional). Paris, Augustin Challamel, $931 \mathrm{p}$.

Naciri-Azzouz A. 2016 - Les variétés arabes de Ghomara : s-saḥəl vs. əğ-ğbəl (la côte vs. la montagne). In : Grigore G. \& Bituna G. (Ed.) Arabic varieties: Far and Wide, Proceedings of the 11th International Conference of AIDA, Bucharest, 2015. Bucuresti, Editura Universitatii din Bucuresti : 405-412.

Natividad E. 1998 - Le dialecte de Chefchaouen. In : Aguadé J., Cressier P. \& Vicente Á. (Ed.) Peuplement et arabisation au Maghreb occidental. Madrid-Zaragoza, Casa de Velázquez-Universidad de Zaragoza : 109-120.

Peña-Chocarro L., Pérez-Jordà G., Morales-Mateos J. \& Zapata L. 2015 - Storage in traditional farming communities of the western Mediterranean : Ethnographic, historical and archaeological data. Environmental Archaeology 20 (4) : 379-389. [En ligne] URL : http://www.tandfonline.com/ doi/abs/10.1179/1749631415Y.0000000004, DOI: 10.1179/1749631415Y.0000000004. [Consulté le 15 juin 2017].

Pereira C. 2012 - L'arabe de Jadu (Libye). In : Barontini A., Pereira C., Vicente Á. \& Ziamari, K. (Ed.). Dynamiques langagières en Arabophonies : variations, contacts, migrations et créations artistiques. Hommage offert à Dominique Caubet par ses élèves et collègues. Zaragoza, Universidad de Zaragoza : 164-199.

Renisio A. 1932 - Études sur les Dialectes berbères des Beni Iznassen, du Rif et des Senhadja du Srair - Grammaire, textes et lexique. Paris, Ernest Leroux, 465 p.

Rosenberger B.1980 - Cultures complémentaires et nourritures de substitution au Maroc ( $\mathrm{XV}^{\mathrm{e}}$ XVIII ${ }^{\mathrm{e}}$ siècle). Annales Économies, Sociétés, Civilisations 35e année (3-4) : 477-503. [En ligne] URL : http://www.persee.fr/doc/ahess_0395-2649_1980_num_35_3_282648 [Consulté le 15 juin 2017].

Samuel D. 1999 - Bread in archeology. Civilisations, Pain, Fours et Foyers des temps passés/Bread, Ovens and Hearths of the past $49: 27-36$. [En ligne] URL : http://www.jstor.org/stable/41229639 [Consulté le 17-08-2017].

Simenel R. 2010 - Beldi / Roumi : une conception marocaine du produit de terroir, exemple des Aït Ba'amran. Hesperis-Tamuda numéro spécial « Patrimoine et patrimonialisation au Maroc » dirigé par M. Berriane $45:$ 167-176.

Vicente Á., Caubet D. \& Naciri-Azzouz A. (Ed.). 2017 - La région du Nord-Ouest marocain : Parlers et pratiques sociales et culturelles. Zaragoza : Prensas de la Universidad de Zaragoza, $357 \mathrm{p}$.

Vicente Á. 2000 - El dialecto árabe de Anjra (norte de Marruecos). Estudio lingüístico y textos. Zaragoza, Universidad de Zaragoza, 285 p.

Vicente Á. 2017 - Les parlers arabes montagnards du Nord du Maroc. Une question d'identité langagière. In : Vicente Á., Caubet D. \& Naciri Azzouz A. (Ed.), La région du Nord-Ouest marocain: Parlers et pratiques sociales et culturelles. Zaragoza, Prensas de la Universidad de Zaragoza : 29-50. 
Ziamari K. \& Barontini A. 2008 - Quelques éléments de description d'un parler Jebli (Ourtzagh, Maroc). Estudios de dialectología norteafricana y andalusí 12 : 43-59.

\section{NOTES}

1. Le Programme Jbala CJB a duré de 2012 à 2014. Le PICS « La Montagne et ses savoirs » de 2013 à 2015, initié par l'IREMAM.

2. Pour nous rendre de Taounil à Msek, nous sommes passés par Had Rouadi, la N16, puis la route qui descend jusqu'à Bni Hadifa, pour finir par la piste.

3. Voir Maghdad 1993.

4. Mission effectuée à l'été 2012 et présentée aux Journées de Tétouan d'octobre 2012. Voir El Jettari 2017. Sur Msek voir aussi Caubet 2016 et 2017.

5. Voir Caubet 2016.

6. Soupe marocaine de rupture du jeûne pendant Ramadan.

7. Mouliéras renvoie au tome $1^{\mathrm{er}} \mathrm{du}$ Maroc Inconnu (1895 :55), où il explique que l'arguel est « un arbuste de la taille du lentisque. Son fruit, semblable à l'arachide est moulu; la farine, ainsi obtenue, sert à faire du pain, du couscous et de la zemmita (farine provenant de la graine torréfiée qu'on détrempe dans l'eau pour la manger)... ». Voir plus loin le terme zemmita.

8. Salem Chaker, communication personnelle.

9. Voir URL : http://saveursdetajine.blogspot.fr/2013/06/zemmita.html. Consulté le 25 février 2017.

10. Voir URL : http://artcuisinemarocains.blogspot.fr/2009/09/zamita-marocaine.html. Consulté le 25 février 2017. C'est aussi le titre d'une chanson d'un des groupes de la scène alternative marocaine, Hoba Hoba Spirit, voir le clip Zemmita réalisé par Hicham Lasri, URL: https:// www.youtube.com/watch?v=rZ5XmJSM9IE. Consulté le 25 février 2017.

11. Voir par exemple URL : https://www.youtube.com/watch?v=CaCNv9iEofU. Consulté le 25 février 2017.

12. La ville s'appelle aujourd'hui Ghazaouet, port algérien très proche de la frontière marocaine.

13. Voir aussi Caubet 2016.

14. Merci à Bernard Caron de ses indications pour cerner cette intonation.

15. Voir les préverbes de l'inaccompli la, ‘a dans exemple Vicente et al. (Ed.) 2017.

16. L'essentiel des traits sont recensés dans Caubet 2017. C'est grâce à elle, ancienne étudiante de Simon Lévy, que nous avons pu accéder à cette région et à ces familles. Merci à elle !

\section{RÉSUMÉS}

La préparation des farines et des pains à partir de céréales, constitue une activité-clef en Méditerranée, zone de l'origine de la domestication des blés, de l'orge, du petit épeautre, du seigle et de l'avoine. Les céréales sont utilisées sous forme de semoules, de pâtes, de farines, de pains levés ou non levés depuis des temps anciens. Notre démarche transdisciplinaire ethnobotanique et linguistique s'attache aux discours et aux termes cherchant à mieux saisir les usages des différentes formes de parlers, et à traduire ce que les groupes sociaux rencontrés attribuent comme signification aux aliments préparés et à la nature des plantes qui les 
composent. Ces études ethnobotaniques et linguistiques dans le Rif ouvrent une voie pour mieux comprendre ces savoirs, à travers l'expression du langage et la nature des échanges entre communautés parlant des dialectes différents.

Le Rif est en effet une situation idéale à la fois pour le linguiste et l'ethnobotaniste pour l'étude des situations d'échanges, de contacts de langues et de techniques alimentaires, aux frontières entre groupes arabophones et berbérophones.

Sur le plan ethnobotanique, ces travaux nous ont permis d'identifier les chaînes opératoires de transformation des céréales en farine, pain ainsi que les modes de cuisson, comportant un lexique très riche et explicitant l'importance de ces produits pour les habitants des deux villages étudiés. Nous discutons cet ensemble de techniques et les usages des produits céréaliers, du levain dans le contexte de leur insertion au sein des champs céréaliers et vergers d'amandiers environnants, et dans l'unité domestique. Il s'agit de données à ce jour inédites pour cette partie du Rif.

The preparation of flours and breads from cereals is a key activity in the Mediterranean region, the area fo origin of the domestication of wheat, barley, small spelled, rye and oats. Cereals have been used in the form of semolina, pasta, flour, leavened and unleavened breads, since ancient times. Our cross-disciplinary ethnobotanical and linguistic approach focuses on discourses and terms that seek to better understand the uses of different forms of speech and to convey what the social groups under study attribute to prepared foods and to the nature of the plants that make them up. These ethnobotanical and linguistic studies in the Rif open the way to better understanding this knowledge, through the expression of language and the nature of exchanges between communities speaking different dialects.

The Rif is an ideal situation for both linguists and ethnobotanists to study exchange situations, language contacts and food techniques, on the borders between Arabic-speaking and Berberspeaking groups.

On the ethnobotanical level, this work allowed us to identify the operating chains of cereal processing into flour, bread and cooking methods, including a very rich lexicon explaining the importance of these products for the inhabitants of the two villages studied. We discuss this set of techniques and the uses of cereal products and leaven, the way they are inserted in the surrounding cereal fields and almond orchards, and in the domestic unit. These data are novel to this day for this part of the Rif.

\section{INDEX}

Keywords : cereals, breads, oven, leaven, Prehilali dialects, Rif, Arabic, Darija, Berber, ethnobotany, dialectology, Jbala

Mots-clés : céréales, pains, four, levain, parlers préhilaliens, Rif, arabe, darija, berbère, ethnobotanique, dialectologie, Jbala

\section{AUTEURS}

\section{DOMINIQUE CAUBET}

Professeur émérite d'arabe maghrébin, INALCO. LaCNAD et Centre Jacques Berque ; 16 Bd. A.

Blanqui, 75013 PARIS

caubet.dominique@yahoo.fr 


\section{YILDIZ AUMEERUDDY-THOMAS}

Directeur de Recherche, CNRS, Centre d'Écologie Fonctionnelle et Évolutive (UMR5175 CEFE) Campus CNRS, 1919 route de Mende - 34293 Montpellier cedex 5, France

yildiz.aumeeruddy-Thomas@cefe.cnrs.fr 